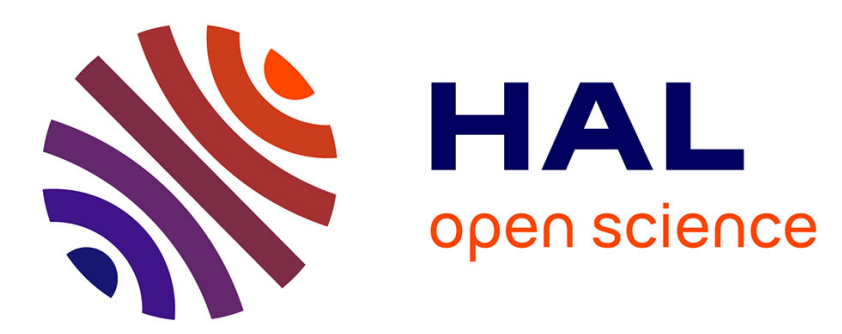

\title{
PFS/MEX observations of the condensing CO south polar cap of Mars
}

M. Giuranna, D. Grassi, V. Formisano, L. Montabone, F. Forget, L. Zasova

\section{To cite this version:}

M. Giuranna, D. Grassi, V. Formisano, L. Montabone, F. Forget, et al.. PFS/MEX observations of the condensing CO south polar cap of Mars. Icarus, 2008, 197 (2), pp.386. 10.1016/j.icarus.2008.05.019 . hal-00499088

\section{HAL Id: hal-00499088 https://hal.science/hal-00499088}

Submitted on 9 Jul 2010

HAL is a multi-disciplinary open access archive for the deposit and dissemination of scientific research documents, whether they are published or not. The documents may come from teaching and research institutions in France or abroad, or from public or private research centers.
L'archive ouverte pluridisciplinaire HAL, est destinée au dépôt et à la diffusion de documents scientifiques de niveau recherche, publiés ou non, émanant des établissements d'enseignement et de recherche français ou étrangers, des laboratoires publics ou privés. 


\section{Accepted Manuscript}

PFS/MEX observations of the condensing $\mathrm{CO}_{2}$ south polar cap of Mars

M. Giuranna, D. Grassi, V. Formisano, L. Montabone, F. Forget, L. Zasova

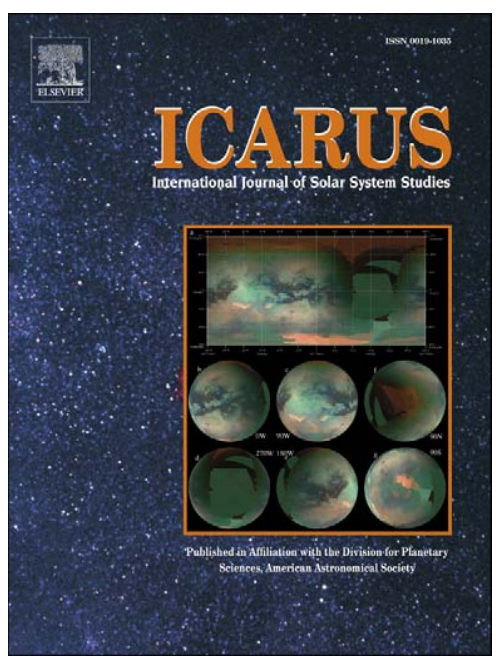

PII: $\quad$ S0019-1035(08)00215-7

DOI: $\quad$ 10.1016/j.icarus.2008.05.019

Reference: $\quad$ YICAR 8693

To appear in: Icarus

Received date: 20 November 2007

Revised date: 4 April 2008

Accepted date: 20 May 2008

Please cite this article as: M. Giuranna, D. Grassi, V. Formisano, L. Montabone, F. Forget, L. Zasova, PFS/MEX observations of the condensing $\mathrm{CO}_{2}$ south polar cap of Mars, Icarus (2008), doi:

10.1016/j.icarus.2008.05.019

This is a PDF file of an unedited manuscript that has been accepted for publication. As a service to our customers we are providing this early version of the manuscript. The manuscript will undergo copyediting, typesetting, and review of the resulting proof before it is published in its final form. Please note that during the production process errors may be discovered which could affect the content, and all legal disclaimers that apply to the journal pertain. 


\title{
PFS/MEX observations of the condensing $\mathrm{CO}_{2}$ south polar cap of Mars
}

\author{
M. Giuranna ${ }^{1,2}$, D. Grassi ${ }^{1}$, V. Formisano ${ }^{1}$, L. Montabone ${ }^{4,5}$, F. Forget ${ }^{5}$, L. \\ Zasova $^{3,1}$
}

1. Istituto di Fisica dello Spazio Interplanetario CNR (IFSI), Via del Fosso del Cavaliere 100, 00133 Roma, Italy.

2. CISAS, Univ. of Padova.

3. Space Research Institute of Russian Academy of Sciences (IKI), Profsojuznaja 84/32, 117810 Moscow, Russia.

4. Department of Physics \& Astronomy, The Open University, Walton Hall, Milton Keynes MK7 6AA, United Kingdom

5. Laboratoire de Météorologie Dynamique du CNRS, Université Paris 6, BP99, 75252, Paris Cedex 05, France

Number of pages: $\quad 62$

Number of figures: 16

Preprint submitted to Icarus

9 Nov 2007

Revised

3 Apr 2008

Accepted

5 May 2008 


\section{Proposed running head:}

The condensing martian south polar cap

\section{Editorial correspondence to:}

\section{Marco Giuranna}

Istituto di Fisica dello Spazio Interplanetario INAF-IFSI,

Via del Fosso del Cavaliere 100

00133 Roma

ITALY

Phone: $\quad+390649934042$

Fax: $\quad+390649934074$

E-mail address: marco.giuranna@ifsi-roma.inaf.it 


\begin{abstract}
The condensing $\mathrm{CO}_{2}$ martian south polar cap and the mechanisms of the $\mathrm{CO}_{2}$ ice accumulation have been studied through the analysis of spectra acquired by the Planetary Fourier Spectrometer (PFS) during the first two years of ESA's Mars Express (MEX) mission. This dataset spans more than half a martian year, from Ls $330^{\circ}$ to Ls $\sim 194^{\circ}$, and includes the southern fall season which is found to be extremely important for the study of the residual south polar cap asymmetry.

The cap expands symmetrically and with constant speed during the fall season. The maximum extension occurs sometime in the $80^{\circ}-90^{\circ}$ Ls range, when the cap edges are as low as $-40^{\circ}$ latitude. Inside Hellas and Argyre basins, frost can be stable at lower latitudes due to the higher pressure values, causing the seasonal cap to be asymmetric. Within the seasonal range considered in this paper, the cap edge recession rate is approximately half the rate at which the cap edge expanded. The longitudinal asymmetries reduce during the cap retreat, and disappear around $\mathrm{Ls} \sim 145^{\circ}$.

Two different mechanisms are responsible for $\mathrm{CO}_{2}$ ice accumulation during the fall season, expecially in the $50^{\circ}-70^{\circ} \mathrm{Ls}$ range. Here, $\mathrm{CO}_{2}$ condensation in the atmosphere, and thus precipitation, is allowed exclusively in the western hemisphere, and particularly in the longitudinal corridor of the perennial cap. In the eastern hemisphere, the cap consists mainly of $\mathrm{CO}_{2}$ frost deposits, as a consequence of direct vapor
\end{abstract}


deposition. The differences in the nature of the surface ice deposits are the main cause for the residual south polar cap asymmetry.

Results from selected PFS orbits have also been compared with the results provided by the martian general circulation model (GCM) of the Laboratoire de Météorologie dynamique (LMD) in Paris, with the aim of putting the observations in the context of the global circulation. This first attempt of cross-validation between PFS measurements and the LMD GCM on the one hand confirms the interpretation of the observations, and on the other hand shows that the climate modeling during the southern polar night on Mars is extremely sensitive to the dynamical forcing.

Keywords: Mars, polar caps; Mars, climate; Ices, condensation; Planetary waves; SRPC 


\section{1 - Introduction}

Despite the large number of studies on the southern spring and summer polar cap recession, with both satellite and ground-based observations, only few studies are available in the literature describing the seasonal progression of the martian south polar cap (SPC) during the fall and winter seasons; in addition, most of them make use of model predictions rather than measurements. This is mainly due to limitations in the visible imaging technique which is used to determine the contrast of albedo between the edge of the seasonal cap and the volatile free soil. Such contrast is practically undetectable where the $\mathrm{CO}_{2}$ cap is dark, as in the south polar cryptic region (Kieffer et al., 2000), and during the polar night (Titus and Kieffer, 2001). Because a large portion of the atmosphere condenses in and sublimes from the seasonal caps in a cyclic way during the year, the frost deposits play a significant role in the regional and global atmospheric circulation. Understanding the nature of the seasonal polar caps is essential if we want to understand the current martian climate with high degree of confidence.

As much as $30 \%$ of the martian atmosphere condenses every year to form polar caps in both hemispheres (Prettyman et al., 2003), inducing large variations of the surface pressure over the entire planet, as first observed by the Viking landers (Hess et al., 1980; Hourdin et al., 1993). At first glance, this phenomenon may seem straightforward, but several previous works on the thermal structure and thermal IR observations of the polar night by the NASA Mars Global Surveyor (MGS) and Mars 
Odyssey missions, suggest that this event is very complex, expecially in the southern hemisphere (Titus and Kieffer, 2001; Banfield et al., 2003; Hinson et al., 2003; Colaprete et al., 2005). Traveling weather systems (transient eddies) are prominent in both hemispheres (J. R. Barnes, 2003; Banfield et al., 2004; Hinson and Wilson, 2002), while the large-amplitude and strongly upper-level disturbances observed for the first time in the northern hemisphere by the Viking Meteorology Experiment (J. R. Barnes, 1980,1981 ) have yet to be observed in the south, despite their prediction by Mars GCM experiments (Barnes et al., 1993). A peculiar and very prominent component of the southern winter circulation, as revealed by the Thermal Emission Spectrometer (TES) and Radio Occultation observations from MGS (Hinson et al., 2003), is a largeamplitude quasi-stationary wave pattern. At lower altitudes this wave can show a fairly strong wavenumber-2 pattern, but at upper altitudes it is dominated by wavenumber 1 . This planetary wave and the complex dynamical processes that occur in the atmosphere during the southern polar night have important effects on the regional climate of the southern hemisphere in winter time (Colaprete et al., 2005).

Whether the $\mathrm{CO}_{2}$ ice accumulation in the polar caps is the result of a direct vapor condensation at the ground (frost), or of atmospheric condensation and precipitation (snow), cannot be easily addressed. The formation of $\mathrm{CO}_{2}$ ice clouds and snowfall in the martian polar night is far from being completely understood, and the direct condensation of the main atmospheric constituent (carbon dioxide) in the polar regions 
during fall and winter is one aspect of the martian meteorology that has no terrestrial counterpart.

In this paper we try to address, at least partially, the lack of information about the condensing $\mathrm{CO}_{2}$ south polar cap of Mars, studying in particular, the progression (advancing or expanding cap) and the recession rate of the $\mathrm{CO}_{2}$ ice deposits towards lower (higher) latitudes and the mechanisms of the $\mathrm{CO}_{2}$ ice accumulation. For this purpose, we have analyzed the PFS spectra acquired during the first two years of the MEX mission. We also provide new insights into the explanation of the residual south polar cap asymmetry which has been proposed by Colaprete et al. (2005).

It is important to note that, in this paper, we focus our interest only on the $\mathrm{CO}_{2}$ cap and the mechanisms of $\mathrm{CO}_{2}$ ice accumulation. The actual extension of the polar ices, including the water ice cap, may be found at different latitudes than those reported for the cap edges, these values being the bound only for the $\mathrm{CO}_{2}$ ice deposits. Hereafter, we will always refer to the polar cap as the $\mathrm{CO}_{2}$ ice polar cap. Moreover, we will use the word "frost" to indicate $\mathrm{CO}_{2}$ that condensed directly onto the surface, with no implications for grain sizes. 


\section{1 - Data set and treatment}

The Planetary Fourier Spectrometer (PFS) for the Mars Express mission is an infrared spectrometer operating in the wavelength range from 1.2 to $45 \mu \mathrm{m}$ separated in two spectral channels, called SWC (short wavelength channel) and LWC (long wavelength channel). These channels cover, respectively, 1.2-5.5 $\mu \mathrm{m}\left(1800-8200 \mathrm{~cm}^{-1}\right)$ and $5.5-45$ $\mu \mathrm{m}\left(220-1800 \mathrm{~cm}^{-1}\right)$. The spectral resolution from the measured monochromatic transfer function is $1.3 \mathrm{~cm}^{-1}$ when no apodization function is applied, and about $2 \mathrm{~cm}^{-1}$ when a Hamming function is applied to the interferograms (this is the case for the present work). The spacecraft is orbiting Mars in a polar orbit having an inclination of $87^{\circ}$, a pericentre altitude of $\sim 250 \mathrm{~km}$ and an orbital period of $7.5 \mathrm{~h}$. The instrument field of view (IFOV) is about $1.6 \mathrm{deg}(\mathrm{FWHM})$ for the $\mathrm{SWC}$ and $2.8 \mathrm{deg}$ (FWHM) for the LWC, which corresponds to a spatial resolution of, respectively, 7 and $12 \mathrm{~km}$ at the pericentre. Additional details about the PFS design, specifications, and operation may be found in Formisano et al., 2005. The spectral and radiometric calibration procedure for both channels has been discussed in detail by Giuranna et al. (2005a, 2005b). Essentially, only the LWC spectra were used in this work, since during the polar night the SWC is "blind": there is no reflected sun light to measure and only negligible thermal emission from the cold $\mathrm{CO}_{2}$ ice (temperature $\sim 145 \mathrm{~K}$ ) in the thermal range of the SWC. The observed LWC radiance depends on several parameters of the atmosphere and surface of Mars, as described by radiative transfer theory: the surface 
temperature and emissivity, the column density of dust and water ice aerosols in the atmosphere, the air temperatures as a function of altitude, the surface pressure, and the column density of water vapor and carbon monoxide. Adopting the very general formalism of Bayesian analysis, an algorithm for the scientific analysis of individual calibrated PFS measurements has been developed, allowing the simultaneous retrieval of the above mentioned parameters. A full description of the adopted method can be found in Grassi et al., 2005. The cases presented in this study were carefully selected imposing a value of chi-square test close to 1 in the selected spectral range of 400-800 $\mathrm{cm}^{-1}$. The Hamming apodization has been applied to all the interferograms used in this study; the effective spectral resolution is thus $\sim 2 \mathrm{~cm}^{-1}$. Typical quality of modeling is presented in figure 1 for different thermal conditions of the atmosphere.

[Fig. 1]

In our retrieval scheme, the entire range $400-1100 \mathrm{~cm}^{-1}$ is used simultaneously for the self-consistent retrieval of the following parameters (state vector elements):
a. surface temperature;
b. air temperature profile (in the indicative range $1-50 \mathrm{~km}$ );
c. integrated silicate dust content;
d. integrated water ice content. 
Noteworthy, the high spectral resolution of PFS allows the detection of several different thermal gradients in the atmosphere, as demonstrated by the effective modeling in the same spectrum of absorbing and emitting Q-branches (lower curves of both panels of figure 1, black arrows).

The general retrieval code adopted for the analysis of PFS calibrated data (Grassi et al., 2005) was designed for a general-purpose usage. In this perspective, an important characteristic is given by its robustness against possible run-time errors due to occasional residual instrumental features in calibrated data and inherent numerical instability of the retrieval inverse problem. Therefore, physical parameters of martian atmosphere computed from PFS data are constrained to lie within realistic ranges. For air temperature, the lower limit was fixed - quite arbitrarily - to the $\mathrm{CO}_{2}$ condensation temperature, assuming an average $\mathrm{CO}_{2}$ mixing ratio of 0.9532 . This choice is motivated by

1. the need to avoid extrapolation of gaseous absorption coefficients outside the temperature range for which they have originally been tabulated;

2. the ambiguity to define a more realistic lower limit without introducing a complete microphysical scheme for the condensation inside the retrieval code.

This constraint implies that, in our retrievals, any physical situation consistent with a supersaturated $\mathrm{CO}_{2}$ atmosphere is actually represented with a temperature profile coinciding with the condensation profile. For this reason, one must be careful in the 
interpretation of the results. Based on the derived vertical temperature profiles alone, one can not distinguish whether it is radiative or adiabatic cooling that is controlling the $\mathrm{CO}_{2}$ condensation in the atmosphere.

In this study we have collected and analyzed a set of 467 orbits acquired during the first two years of PFS activity. This dataset spans more than half a martian year, from $\operatorname{Ls} \sim 330^{\circ}$ to $\operatorname{Ls} \sim 194^{\circ}$, although there are some gaps. For instance, during the whole winter season the orbit geometry is such that we rarely observe latitudes poleward of $60^{\circ}$, making it impossible to compute longitude the cross-sections of the vertical profiles of $\left(T-T_{\text {sat }}\right)$ at polar latitudes that are needed for a comprehensive study of the mechanisms of the $\mathrm{CO}_{2}$ ice accumulation. Some other short Ls ranges are also missing, such as the $39^{\circ}-49^{\circ}$ range. Nevertheless, the data allow a detailed study of the $\mathrm{CO}_{2}$ south polar cap formation rates and mechanisms, especially during the fall season which has been found to be of great importance for the explanation of the residual south polar cap asymmetry.

\section{$1.2-\mathrm{CO}_{2}$ ice clouds and snowfalls: theoretical considerations}

Before showing the vertical structure of the atmospheric temperature above the southern polar cap during the polar night as measured by PFS and discussing the possible implications for the martian climate and the mechanisms of the $\mathrm{CO}_{2}$ ice accumulation, it 
is important to analyze the various processes which lead to cooling and condensation of the atmosphere, and thus to $\mathrm{CO}_{2}$ snowfall. Although it is generally assumed that most of the dry ice condenses directly on the surface, a fraction might also condense in the atmosphere, strongly influencing its radiative properties.

Since $\mathrm{CO}_{2}$ ice clouds essentially form in the polar night, the evidence of their existence has remained theoretical or indirect. It is only recently that a variety of cloud shapes varying over space and time has been observed by the MGS laser altimeter MOLA (Pettengill and Ford, 2000; Colaprete and Toon, 2002; Tobie et al., 2003). The radiative effect of snow would strongly alter the radiative balance of the condensing polar caps and thus the $\mathrm{CO}_{2}$ cycle and the global climate. Forget et al. (1995) showed that the low emission zones observed by the infrared thermal mapper (IRTM) instrument aboard Viking (Kieffer et al., 1976) and by Mariner 9 IRIS (Paige et al., 1990), were likely due to the radiative properties of $\mathrm{CO}_{2}$ snowfalls (falling snow particles or fresh snow deposits). $\mathrm{CO}_{2}$ snowfall has never been directly observed on Mars, although several authors reported circumstantial evidences of a possible $\mathrm{CO}_{2}$ snowstorm (Titus et al., 2001; Ivanov and Muhleman, 2001). Nevertheless, climate simulation with general circulation models (GCMs) showed that a fraction of the total $\mathrm{CO}_{2}$ condensation could take place in the atmosphere, especially when it is dust laden, because of the increased atmospheric emissivity (Pollack et al. 1990). In this case, atmospheric condensation is the result of radiative cooling. Atmospheric condensation may also result from 
adiabatic cooling in upward motions (Forget et al., 1998). The change in temperature with expansion or compression is called the adiabatic process. When a parcel of air changes its altitude, its pressure changes; consequently, its temperature changes as well. If an air parcel rises, it expands, and the temperature decreases (adiabatic cooling); if an air parcel falls, it compresses and the temperature increases (adiabatic heating). This process can be very efficient locally.

\section{Radiative cooling}

During the polar night, the radiative balance of surface $\mathrm{CO}_{2}$ is negative, barring an extraordinarily warm atmosphere. The radiative budget is reduced to cooling due to the emission of thermal infrared radiation. When the surface is cooled to the frost point, its temperature is kept constant by the latent heat released by surface condensation, while the atmosphere is radiatively warmed by the surface infrared emission. Gierash and Goody (1968) and, more recently, Paige (1985) pointed out that the radiative equilibrium of a surface plus atmosphere column is always colder than the condensation temperature profile. Thus, in the polar night, the atmospheric column may rapidly cool and condense out. The latent heat released by condensation would then balance the radiative cooling to keep the temperature at the frost point value everywhere in the atmosphere (actually, everywhere that condensation is taking place). As Forget et al. (1998) have shown that, in such conditions, the cooling and condensation rates depend 
only on the radiative properties (emissivity and transmittivity) of the ground and the atmosphere; these are lowest for a clear atmosphere $(\tau=0)$ above a blackbody surface $(\varepsilon=1)$ : only $3 \%$ of the $\mathrm{CO}_{2}$ condenses in the atmosphere in that case. The presence of dust strongly increases the emissivity of the atmosphere and thus the cooling rate. The radiative properties of the surface also affect the equilibrium of the atmosphere: when the cap surface emissivity is lower than one, the infrared heating of the atmosphere by the ground is reduced, and the atmospheric condensation increases. With surface emissivity $\varepsilon=0.7$ and $\tau=1$, the fraction of $\mathrm{CO}_{2}$ condensing in the atmosphere reaches $25 \%$ (Forget et al., 1998).

\section{Adiabatic cooling}

The atmospheric circulation affects the thermal structure of the polar atmosphere. It is well known that the atmosphere transports heat from the insolated latitudes to the polar regions (Pollack et al., 1990; Hourdin et al., 1995). In addition, the circulation can locally warm or cool adiabatically the atmosphere. Cooling occurs in upward motions. Forget et al. (1998) have simulated both the heat advection and the large-scale adiabatic effects during the southern winter $\left(\mathrm{Ls}=120^{\circ}\right)$, using a $1 \mathrm{D}$ version of the LMD martian GCM radiative transfer code (Hourdin et al., 1993 and 1995) with 25 layers. As a result, they found that, according to the model, the atmospheric condensation rate reaches more than ten times the value corresponding to the radiative cooling alone in two special 
condensation zones around $75^{\circ} \mathrm{S}-135^{\circ} \mathrm{W}$ and $67.5^{\circ} \mathrm{S}-20^{\circ} \mathrm{W}$. These areas are created by a dynamic mechanism originating around two low-pressure zones located at about $60^{\circ} \mathrm{W}$ and $300^{\circ} \mathrm{W}$ and related to the Argyre and Hellas basins. This is a typical effect of the southern winter stationary waves which are forced by topography (Hollingsworth and Barnes, 1995; Banfield et al., 2003; Hinson et al., 2003).

\section{2 - The Residual South Polar Cap (RSPC)}

The first spacecraft observations of the south residual polar cap of Mars were obtained by the Mariner 9 orbiter during the martian southern summer season, in 1971-1972. Viking orbiter observations obtained three Mars years later (in 1977) have first shown that residual carbon dioxide ice was present at the south polar cap (Kieffer, 1979). As shown in Figure 2b, PFS observations of the residual cap clearly show the spectral characteristics of $\mathrm{CO}_{2}$ ice and have been used to study its composition in terms of $\mathrm{CO}_{2}$ grain size, and dust and water ice contamination (Hansen et al., 2005). In fact, the carbon dioxide survives all year long in the south residual cap (Bibring et al., 2004; Hansen et al., 2005; Douté et al., 2005); this behavior is surprisingly different from what has been observed in the north, where the $\mathrm{CO}_{2}$ completely sublimes by the beginning of the summer season. Paige and Ingersoll (1985) calculated the annual radiative budgets and infer the annual $\mathrm{CO}_{2}$ frost budgets, showing that the $\mathrm{CO}_{2}$ 
sublimation rates are much smaller in the south than in the north, due to the higher albedo of the RSPC relative to the north cap. This difference in albedo, added to the difference in subsurface heat conduction - which is rather high on the residual north cap and close to zero on the RSPC where the temperature is constant all year long (Jakosky and Haberle, 1990) - explains why the north and south residual polar caps are so different. What still has to be explained is the position of the south permanent cap. Figure 2a shows the RSPC in a mosaic of three OMEGA images. The residual south polar cap is approximately $400 \mathrm{Km}$ across and the cap center is displaced by about $3^{\circ}$ from the geographic pole. Mars eccentricity or local topographic irregularities cannot straightforwardly account for this asymmetry, nor the above mentioned energy balance models can reproduce it. In fact, this has been a major issue in Mars science for many years. A possible explanation has been recently suggested by Colaprete et al. (2005). In this work, we provide new insights for the explanation of the RSPC asymmetry.

[Fig. 2a] [Fig. 2b]

\section{$3-\mathrm{Ls}=0^{\circ}-25^{\circ}:$ the early-fall south polar cap}

Direct observations of frost deposition are generally difficult to obtain due to the fact that most of the frost at high latitudes is deposited when the region is in darkness. 
However, radiometry data obtained by MOLA suggest that frost at the higher latitudes starts to form on the surface before the end of (southern) summer, when the subsolar point is approaching the equator but the pole is still illuminated (M. T. Zuber and D. E. Smith, 2003). Indeed, in the range of Ls examined in this section, soon after the end of summer $\left(\mathrm{Ls}=0^{\circ}-25^{\circ}\right.$ ), the asymmetry observed in the residual cap (figure 2a) has completely vanished; the cap appears symmetric and extends already up to $-70^{\circ}$ latitude, as we can see from the mosaics of tracks of the surface temperatures in figure 3 (top-left panel). The colored symbols used in the maps have fixed size and are not representative of the actual PFS footprints.

[Fig. 3]

The MEX orbit is elliptical, with a precession of its pericentre. In early fall $\left(\mathrm{Ls}=20^{\circ}\right)$ the pericentre is around $-55^{\circ}$ latitude and the cap edges are close to $-70^{\circ}$ latitude, where the PFS footprint is less than $20 \mathrm{Km}$. At Ls $=50^{\circ}$ the pericentre is close to the south pole, while the cap edges are around $-50^{\circ}$ latitude, which corresponds to a footprint of $\sim 30 \mathrm{Km}$. In early winter $\left(\mathrm{Ls}=110^{\circ}\right)$, the pericentre is close to the equator, and the PFS footprint at the polar edges (around $-50^{\circ}$ latitude) is $\sim 40 \mathrm{Km}$. At $\mathrm{Ls}=190^{\circ}$, the pericentre is close to the North pole and the footprint is now close to $350 \mathrm{Km}$. The most 
favorable condition for cap edge detection occurs at Ls $\sim 30^{\circ}$, where the pericentre is essentially at the same latitude as the polar edges $\left(-65^{\circ}\right.$; see Giuranna et al., 2007a).

Whether the $\mathrm{CO}_{2}$ ice at these values of Ls is frost or snow cannot be addressed only on the basis of the surface temperatures. In both cases these temperatures are similar, forced by the vapor pressure equilibrium with the atmosphere (Giuranna et al., 2007a). In principle, a first discrimination may be done on the basis of the grain sizes. $\mathrm{CO}_{2}$ snow grain sizes are expected to be smaller than those derived by a continuous vapor deposition. Unfortunately, when the cap is not illuminated (polar night or night side of a sol) the PFS Short Wavelength Channel, which has shown great capabilities of retrieving ice grain sizes with its NIR spectra (Hansen et al., 2005; Giuranna et al., 2007b), is unusable. Nevertheless, a detailed study of the temperatures in the atmospheric column over the cap can be done using the thermal infrared radiation measured by the PFS Long Wavelength Channel, allowing us to answer the question straightforwardly. Indeed, if the atmospheric temperatures are always above the $\mathrm{CO}_{2}$ condensation temperature in the whole column, the snowfall hypothesis can be reasonably rejected. As anticipated in section 1.1, the algorithm developed by Grassi et al., 2005, allows, among other things, the simultaneous retrieval of surface temperature, integrated content of water ice and dust suspended in the atmosphere, and, most important for the issue we want to address, the air thermal fields up to an altitude of about $50 \mathrm{Km}$. By comparing the measured vertical temperature profiles with the $\mathrm{CO}_{2}$ 
condensation temperature at different altitudes we can establish if, where and at which altitudes $\mathrm{CO}_{2}$ snow falls may be allowed.

Our results show that, at these values of Ls, the south polar cap essentially consists of $\mathrm{CO}_{2}$ frost deposits condensed directly on the surface. A typical situation is depicted in figure 4, where we show the study for a single representative orbit. Orbit number is 287 and Ls is $17^{\circ}$, right in the middle of the Ls range examined in this section. The surface temperatures (top-right panel) reveal the presence of $\mathrm{CO}_{2}$ ice up to $-70^{\circ}$ latitude, which is essentially frost. This can be confirmed by monitoring the vertical profiles of $\Delta \mathrm{T}_{\text {sat }}(\mathrm{z})$ $=\mathrm{T}(\mathrm{z})-\mathrm{T}_{\text {sat }}(\mathrm{z})$ (where $\mathrm{z}$ is the atmospheric altitude): as shown in the bottom panel of figure 4 , the atmospheric temperatures are far above the saturation value in the entire column and at every latitude, with only one exception in a narrow region centered around $-85^{\circ}$ latitude (mid-right panel; this is one of the very few $\Delta \mathrm{T}_{\text {sat }}<0$ occurrences in the Ls range here examined).

[Fig. 4]

If we repeat this study for the whole set of orbits, and thus for the whole Ls $=0^{\circ}-25^{\circ}$ range, we can confirm that the situation described for orbit 287 is indeed the typical early-fall south pole situation. In figure 5 we show the $\Delta \mathrm{T}_{\text {sat }}=\mathrm{T}-\mathrm{T}_{\text {sat }}$ for a longitude cross-section at $-85^{\circ}$ latitude as a function of altitude: the $\mathrm{CO}_{2}$ condensation in the 
atmosphere is forbidden almost everywhere, the atmospheric temperature being always few or several degrees above the $\mathrm{CO}_{2}$ condensation temperature at any altitude.

Consequently, we can conclude that the early-fall south polar cap consists of $\mathrm{CO}_{2}$ frost deposits that directly condense on the surface. Atmospheric condensation is allowed only occasionally and only for latitudes $\leq-84^{\circ}$. Thermal inversions are present almost everywhere, especially in most of the eastern hemisphere, and centered around $5 \mathrm{Km}$ above the surface.

[Fig. 5]

\section{$4-\mathrm{Ls}=25^{\circ}-38^{\circ}$ : early/mid-fall cap and evolution of edges from fall to winter}

Unfortunately, as can be seen in figure 3 (top-right panel), during this Ls range we observed latitudes poleward of $-80^{\circ}$ only twice, during orbit 371 and 378 . It is, therefore, impossible to study in detail the mechanisms of the $\mathrm{CO}_{2}$ ice accumulation as we did in the previous section for the early-fall season, by computing longitudinal cross-sections of $\mathrm{T}-\mathrm{T}_{\text {sat }}$ at polar latitudes (Fig. 5). Nevertheless, repeating the same study as in figure 4 for these two orbits, and for all the other orbits within this Ls range, we found that, at least for the observed latitudes and longitudes, no $\mathrm{CO}_{2}$ condensation zones were present anywhere in the atmosphere, suggesting a scenario similar to the one 
described in the previous section, with a cap mainly consisting of $\mathrm{CO}_{2}$ frost deposits directly condensed on the surface.

The south polar cap edges are advancing towards lower latitudes throughout the autumn. During the $25^{\circ}-38^{\circ} \mathrm{Ls}$ range the edge of the cap appears symmetric, located near $-60^{\circ}$ latitude. If we compare the first two panels of figure 3 (the top-left and the top-right panels) we see that the cap edge has grown by $10^{\circ}$ of latitude in about 15 degrees of Ls. The PFS south pole observations pause at Ls $=38^{\circ}$ and start again at Ls $=$ $50^{\circ}$, when a large amount of polar spectra have been acquired up to Ls $=70^{\circ}$. At that Ls the cap is close to its maximum observed extension, with $\mathrm{CO}_{2}$ ice deposits as equatorward as $-40^{\circ}$ latitude (figure 3 , middle-left panel). This is exactly the latitude we would get if we assume a constant progression rate over the whole $25^{\circ}-70^{\circ}$ Ls period. These simple considerations lead us to a first estimation of the cap progression speed during the expansion phase, that is most of the fall season, until Ls $\sim 70^{\circ}$ when the cap slacks its expansion and eventually starts to shrink. A detailed tracking of Mars' south polar cap edges has been recently performed by Giuranna et al. (2007a), exploiting the rapid increase (decrease) of surface temperature that occurs when $\mathrm{CO}_{2}$ ice is removed (deposited). The dataset used in Giuranna et al. (2007a) is exactly the same as the one used for the present study. By using a technique for the cap edge detection that keeps into account the actual $\mathrm{CO}_{2}$ partial pressure at the surface (upon which the actual $\mathrm{CO}_{2}$ condensation temperatures at the surface depend), they have shown that the cap actually 
expands with a constant speed of about $10^{\circ}$ of latitude per 15 degrees of Ls during most of the fall season (until Ls $\sim 70^{\circ}$ ) at every longitude. The latitude of the edge of the south polar cap as a function of season is shown in figure 6 . The maximum extension of the south polar cap occurs sometime in the $80^{\circ}-90^{\circ}$ Ls range, when the cap edges are as low as $-40^{\circ}$ latitude. The $\mathrm{CO}_{2}$ ice south polar cap will then recede with a constant speed of $\sim 5^{\circ}$ of latitude every 25 degrees of Ls during the whole winter. Interesting, inside the Hellas and Argyre basins (red triangles in figure 6) the $\mathrm{CO}_{2}$ condensation temperature is several degrees higher than that of the rest of the south polar region according to the Clausius-Clapeyron's law due to the higher pressure. Therefore the $\mathrm{CO}_{2}$ frost can be stable at lower latitudes (and higher temperatures), extending up to $30^{\circ}$ latitude (occasionally even at lower latitudes), causing the cap to be asymmetric. Thus, the two major martian basins appear to be responsible for a south polar cap asymmetry.

[Fig. 6]

A comparison with MGS Thermal Emission Spectrometer observations during the two previous martian years (Titus, 2005) suggests that this asymmetry repeats every year (solid lines in figure 6), although significant differences are present between the results of the two experiments, especially in the $50^{\circ}-100^{\circ}$ Ls range, with PFS data showing a 
much more extended cap. As Giuranna et al. (2007a) pointed out, these differences may be either due to actual interannual variability or, more likely, caused by differences in the dataset and the techniques adopted for the cap edge detection (see Kieffer et al., 2000, for a description of the TES data analysis technique).

The longitudinal asymmetries decrease during the cap retreat, as the $\mathrm{CO}_{2}$ ice/frost sublimates, and disappear completely around Ls $\sim 145^{\circ}$. Afterwards, the cap continues to recede symmetrically for the rest of the winter season.

\section{5 - Ls $50^{\circ}-70^{\circ}$ : two distinct regional climates}

\section{1 - Two distinct regional climates close to the pole}

In the two previous sections we have shown that during the first half of the southern fall the cap consists of $\mathrm{CO}_{2}$ frost deposit, the atmospheric temperatures being always above the $\mathrm{CO}_{2}$ condensation temperature in the whole column, at every latitude and longitude (with few sporadic exceptions in the western hemisphere). A detailed study of the vertical temperature profiles above the cap, retrieved by the PFS LWC spectra, reveals that the mid-late fall season offers a much more complex and interesting scenario.

In figure $7 \mathrm{a}$ we show the $\Delta \mathrm{T}_{\mathrm{sat}}=\mathrm{T}-\mathrm{T}_{\mathrm{sat}}$ for a longitudinal cross-section in a narrow stripe of latitude $\left(84^{\circ}-86^{\circ} \mathrm{S}\right)$ as a function of altitude. In this selected season range $\left(50^{\circ}\right.$ 
$-70^{\circ} \mathrm{Ls}$ ) two distinct regional climates clearly characterize the south polar cap. $\mathrm{CO}_{2}$ snow falls are now allowed, but they occur exclusively in the western hemisphere. In the eastern hemisphere (more precisely, in the $30^{\circ}-210^{\circ}$ east-longitude range), the whole atmospheric column is several degrees above the saturation temperature; here, the direct vapor deposition is the only allowed mechanism for the $\mathrm{CO}_{2}$ ice accumulation in the South polar cap. In the western hemisphere, instead, atmospheric condensation is allowed in the entire 0-30 km column; here, surface ice accumulation may be dominated by precipitation. This is especially true in two particularly cold regions, located around $260^{\circ} \mathrm{E}$ and $320^{\circ} \mathrm{E}$ longitude, where the $\mathrm{CO}_{2}$ condensation is allowed to a large extent in the atmosphere, from the surface up to $20 \mathrm{~km}$ and $30 \mathrm{~km}$, respectively. It is very interesting to note that the coldest region (blue arrow in figure 7a) is located right around the center of the south residual cap (blue dotted line in Fig. 7b; the south residual cap extends roughly from $250^{\circ} \mathrm{E}$ to $45^{\circ} \mathrm{W}$ longitude, and is centered around $320^{\circ}$ E longitude).

[Fig. 7a] [Fig. 7b]

Note that both the high-condensation rate zones predicted by the 1D GCM simulations described in section 1.2 are in agreement with the two cold regions observed by PFS. In particular, the coldest one, which is predicted by the models to be around $340^{\circ} \mathrm{E}$ 
longitude, is only $10^{\circ}$ longitude away from the one we have found in the present work. Note also that the warmest region is located in the $40^{\circ}-90^{\circ}$ east-longitude range, i.e. right below the Hellas basin. An increase of the atmospheric temperatures below Hellas and poleward of $-60^{\circ}$ latitude is also predicted by the Mars GCM model, as a result of dynamical forcing by the largest southern impact basin (Forget et al., 2006; EMCD 4.1 pre-computed version, available online at http://www-mars.lmd.jussieu.fr).

Figure 8 shows several PFS temperature retrievals in the "cold" hemisphere (solid curves) and in the "warm" hemisphere (dashed curves) at $85^{\circ} \mathrm{S}$ latitude for different longitudes, in the range of areocentric longitude $50^{\circ}-70^{\circ}$. The two hemispheres show very different profiles at this time of year. As it has already been pointed out using Figure $7 \mathrm{a}$, in the cold hemisphere we have areas (longitudes) where the atmospheric temperature is close to (but above) the $\mathrm{CO}_{2}$ condensation temperature (black solid curves), and areas where $\mathrm{CO}_{2}$ condensation in the atmosphere is allowed in a variety of altitudes (colored solid curves). Figure 8 illustrates several cases where condensation is allowed from the ground up to $10 \mathrm{~km}$ (blue curve), $15 \mathrm{~km}$ (orange curve) and $20 \mathrm{~km}$ (red curve); we may also have condensation areas located around $15 \mathrm{~km}$ (yellow and green curves) and, occasionally, from $\sim 15$ to $30 \mathrm{~km}$ (cyan curve).

[Fig. 8] 
In the warm hemisphere $\left(30^{\circ}-210^{\circ}\right.$ east-longitude) the atmospheric temperatures are always far above the $\mathrm{CO}_{2}$ condensation temperatures and three types of profiles can be distinguished. Moving eastward we observe the following situations.

a) The warmest region (red-dotted curves in figure 8 ) is located in the $40^{\circ}-90^{\circ} \mathrm{E}$ longitude range which corresponds essentially to the margins of the Hellas basin. The whole atmospheric column is warm (at least $10-15 \mathrm{~K}$ above the $\mathrm{CO}_{2}$ condensation temperature), with strong temperature inversions around 5-10 km altitude.

b) A wide area $\left(80^{\circ}-180^{\circ} \mathrm{E}\right.$ longitude) shows weaker inversions at $5-10 \mathrm{~km}$ and a colder atmospheric column (light-blue-dotted curves in figure 8). These are the "typical" profiles in the warm hemisphere.

c) A narrow area at the eastern limits of the warm region $\left(180^{\circ}-210^{\circ} \mathrm{E}\right.$ longitude) shows strong temperature inversions around $5 \mathrm{~km}$ altitude and a higher temperature gradient (and consequently lower temperatures at 30-35 km altitude).

In all these cases, the polar warming starts at $30-35 \mathrm{~km}$ altitudes.

The residual cap lies in the cold region, where $\mathrm{CO}_{2}$ snowfalls occur. The black dotted line in figure $7 \mathrm{~b}$ separates the two hemispheres as defined in figure $7 \mathrm{a}$, where the black double-arrow indicates the extension and the edges of the warm region. These two distinct regional climates produce differences in the nature of the surface ice deposits during the whole second half of the fall season, and are the main responsible for the 
residual south polar cap asymmetry. The scenario depicted in figure 7 a starts around Ls $=50^{\circ}-55^{\circ}$, and remains essentially unaltered until the end of the Autumn. The only exception is that, after $\mathrm{Ls}=70^{\circ}$, the atmospheric temperatures above the polar regions are generally lower, therefore $\mathrm{CO}_{2}$ condensation in the atmosphere occurs in a wider area and, occasionally, also in the western hemisphere (see section 7). It is interesting to understand how this very peculiar scenario with two distinct regional climates close to the south pole can take place; we will show in the following section that the observations from PFS confirm that it is essentially the result of dynamical forcing by the largest southern impact basins, Argyre and Hellas.

\section{2 - Hadley circulation, winds and planetary waves from PFS nadir observations}

The vertical temperature profiles inferred from measurements of thermal emission by instruments aboard orbiting spacecrafts, such as the MEX/PFS LW Channel, represent the best available way to get indirect information on the atmospheric circulation. These data can provide more complete spatial and temporal coverage of Mars than direct measurements from landers or rovers on the surface, and can be used to infer winds. The vertical variation of the horizontal wind components (i.e., the wind shears) can be computed from global temperature fields, using the geostrophic or higher-order approximations (Zurek et al., 1992), while the zonal-mean (longitudinal average) meridional and vertical winds can be calculated by combining the thermodynamic 
energy and mass continuity equations. The zonal-mean component of the zonal wind can be derived in a rather straightforward way from the observed temperature field, by using the thermal wind relation (see, e.g., Holton, 1979; Andrews et al., 1987):

$$
\frac{\partial}{\partial z}\left[\left(f+\frac{\bar{u} \tan \theta}{a}\right) \bar{u}\right]=-\frac{R}{m a} \frac{\partial \bar{T}}{\partial \theta}
$$

where $\bar{T}$ is the zonal mean temperature, $\bar{u}$ is the zonal mean eastward wind component, $\theta$ is the latitude, $f$ is the Coriolis parameter, $a$ is the planetary radius, $R$ is the gas constant, $z$ is the relative pressure, and $m$ is the atmospheric mean molecular weight. This relation cannot be used close to the equator; here the gradient winds cannot be computed since the assumed force balance is not valid at low latitudes (Holton, 1979). The computation of the gradient winds requires a boundary condition for the zonal wind speed. It is assumed here that $\bar{u}=0$ at the planetary surface and (1) is integrated upward, yielding the baroclinic component of the wind field. Any nonzero surface wind must be added to the result to obtain the total wind speed. On the Earth, the contribution of the surface wind to the total wind in the mid-troposphere is usually small, and integration of (1) with $\bar{u}=0$ at $z=0$ provides a good approximation to $\bar{u}(z)$. It is reasonable to expect this approximation to be valid on Mars (Leovy, 1969 and 2001). Although the calculation of $\bar{u}$ from (1) is simple in principle, care must be taken 
because of the required numerical differentiation of the temperature with respect to latitude. For this purpose the temperatures were smoothed in latitude using a $4^{\circ}$ wide window centered on latitudes evenly spaced by $2^{\circ}$.

The mean meridional thermal structure as retrieved from the PFS LWC thermal spectra in the $50^{\circ}-70^{\circ}$ Ls range, and the corresponding zonal mean eastward wind field derived from thermal gradient wind balance (1) are shown in figure 9. For the temperature cross-section in the meridional plane (figure 9a) an adequate coverage in longitude and latitude is ensured by the large set of available PFS observations. Air temperatures from the individual retrievals were binned in latitude (two-degrees wide bins) and then averaged to obtain mean air temperature fields. This has been done for each of the pressure levels at which temperatures were retrieved. Generally speaking, the changes in the atmospheric temperature fields and, consequently, in the thermal wind fields, are a consequence of the different solar heating with seasons. In the winter hemisphere we have the minimum solar heating at the corresponding solstice, when the atmospheric temperatures reach minimum values. The highest atmospheric temperatures occur instead during the summer solstice. Despite this, we observe from figure 9a that the temperature field shows a local maximum at an altitude of about $40 \mathrm{~km}$ in the polar night hemisphere, i.e. the temperature field is warmer than the thermodynamic equilibrium. This polar warming is dynamic: it is caused by adiabatic heating produced by the mean meridional residual circulation (Zurek, 1992). This type of circulation can 
be described in terms of an ascending branch, near $30^{\circ}$ latitude in the warm northern hemisphere, and a descending branch near $60^{\circ}$ latitude in the cold southern hemisphere. It is similar to Earth's Hadley circulation, with the difference that the cells are much stronger and displaced far from the equator (Leovy, 2001).

[Fig. 9a]

[Fig. 9b]

The local temperature maximum in the latitude belt $50-60^{\circ}$ of the winter hemisphere shown in figure 9a results from adiabatic warming associated with the descending branches of the Hadley cell. Although the Hadley circulation on Mars is stronger near the solstices, we observe that it is already prominent during the mid-late southern fall season. The resulting steep gradient gives rise to strong zonal eastward winds. It is well known (Zurek et al., 1992; Conrath et al., 2000; Leovy, 2001) that the general circulation of Mars is characterized by strong eastward winds in the winter hemisphere. Our results show that this is also true for the mid-late fall season $\left(50^{\circ}-70^{\circ}\right.$ Ls range $)$, as Figure $9 \mathrm{~b}$ shows. The strongest winds, as expected, are in the hemisphere of the polar night, where the polar vortex is well developed. Indeed, in this region the horizontal temperature gradient between the local maximum and the cold polar night is steeper than in any other region. Winds reach $110 \mathrm{~m} / \mathrm{s}$ at roughly $55^{\circ} \mathrm{S}$ and $0.1 \mathrm{mbar}$. The 
interaction of this flow with zonally varying topography excites stationary waves, which take the form of planetary (Rossby) waves for forcing at the largest horizontal scales.

Evidence of planetary-scale, wavelike disturbances in Mars' winter atmosphere was first provided by Mariner 9 and Viking spacecraft observations (J. L. Hollingsworth and J. R. Barnes, 1996). Possible sources of the wave activity were suggested to be dynamical instabilities (e.g. barotropic and/or baroclinic instabilities), and quasistationary planetary waves, i.e. waves that arise predominantly via zonally asymmetric surface properties. Banfield et al. (2003) have characterized the global structure and seasonal evolution of these waves by analyzing nadir observations from the MGS Thermal Emission Spectrometer, and have confirmed that these are stationary planetary waves with significant amplitudes at zonal wavenumbers $\mathrm{s}=1$ and 2 . The $\mathrm{s}=1$ stationary planetary wave is likely the dominant mechanism for eddy meridional heat transport for the southern winter. Hinson et al. (2003) provided similar results to those of Banfield et al. (2003) by combining MGS Radio Science and TES limb data. In addition to an enhancement of the vertical resolution and an extension of the vertical range, they obtained a unique measure of the geopotential field as well as the implied meridional winds and the poleward eddy heat flux. According to Hinson et al. (2003), the geopotential field of the $\mathrm{s}=2$ component is "barotropic" in character, while the $\mathrm{s}=$ 1 component propagates vertically, as reflected by a westward tilt with increasing height in the geopotential and temperature fields and a net poleward eddy flux. These 
upwelling and downwelling motions in the atmosphere cause expansions and compressions of air masses which, in turn, effect variations of temperature. Thus, an indirect way to detect and characterize such a complex dynamical process is to study the effects on the atmospheric temperatures.

A general view of the planetary wave that characterizes the south polar region of Mars during the mid-late fall season as seen by PFS is given in figure 10, where we show the atmospheric temperature of the polar region at an altitude of $40 \mathrm{~km}$. We remark here that the data used to produce the results in figure 10 where highly selected in local time, in order to avoid diurnal/tidal effects. All the measurements in the latitude belt $50^{\circ}-60^{\circ}$ $\mathrm{S}$ are within the narrow range 7:00-7:30 local time. Above $20 \mathrm{~km}$ altitude, the south polar circulation is controlled by a wavenumber-one quasi-stationary planetary wave that produces longitudinal variation in the circulation and climate. The black dotted line in the figure separates the polar region into two parts. One has a strong increase of the atmospheric temperature around the latitude belt $50^{\circ}-60^{\circ} \mathrm{S}$, which is associated with adiabatic warming produced by downwelling of air masses. The other part is where upwelling occurs, resulting in adiabatic cooling.

[Fig. 10] 
The eastward phase shift with increasing distance from the equator observed by Hinson et al. (2003) may explain the correlation of the cold and warm hemispheres in figure 10 with respect to those found at $85^{\circ} \mathrm{S}$. In fact, the longitudinal thermal asymmetries between the western and eastern hemispheres observed at southern high latitudes (figure 7a) are a result of this planetary wave that is excited primarily by the middle-latitude surface topography, namely the basins of Hellas and Argyre.

Our results should be viewed in the context of the broad description of stationary waves derived from TES nadir measurements by Banfield et al. (2003). Within the southern hemisphere they report significant amplitudes at both $\mathrm{s}=1$ and 2 throughout the fall and winter seasons. There is a notable difference in the seasonal evolution of the two modes. The $\mathrm{s}=1$ amplitude has a distinct maximum around southern winter solstice $\left(\mathrm{Ls}=90^{\circ}\right)$, while the $\mathrm{s}=2$ amplitude is relatively weak at the solstice and strongest in midfall and midwinter. For a better characterization of the planetary wave, such as amplitude and phase of the component with zonal wavenumber one, we analyzed in detail each of the vertical temperature profiles inside the area of interest. The results are well summarized in figure 11, where we show the vertical temperature profiles along a single representative PFS orbit. The orbit is number $695\left(\mathrm{Ls}=70^{\circ}\right)$, where the PFS observations cross the entire polar cap, being half in the warm hemisphere, and half in the cold one. The orbit starts from latitude $40^{\circ} \mathrm{N}$ and moves southward; it crosses the Hellas Basin, right over its center $\left(\sim 70^{\circ} \mathrm{E}\right.$ longitude $)$, passes close to the south pole at a 
minimum latitude of $86.67^{\circ} \mathrm{S}$, and then moves northward up to $30^{\circ} \mathrm{S}$ latitude. Strong differences in the atmospheric temperature in the two hemispheres are clearly visible. Once again, these differences are not due to differences in local times, but are a consequence of the dynamic processes associated with the planetary wave. The increase of temperatures in the whole atmospheric column observed near the southern edge of the Hellas basin cannot be attributed (at least not exclusively) to the descending branch of the Hadley cell. An extra heating is due to downward motions and high pressure zones associated with the planetary wave. Atmospheric cooling is present in the other hemisphere, due to upward motion and low pressure zones, and the effect of the descending branch of the Hadley circulation is essentially canceled out. This will be also confirmed by the comparison with GCM model results in section 5.3. We note here that, if we ignore the possible effects caused by differences in the soil albedo and thermal inertia, for a flat, perfectly spherical planet, the effect of the Hadley circulation should be longitude-independent, i.e. the same for a given latitude at a given local time.

[Fig. 11]

The phase of $s=1$ is defined as the east longitude of the maximum in temperature. The wave achieves its largest amplitude of about $10-15 \mathrm{~K}$ near $45 \mathrm{~km}$ altitude and $55^{\circ} \mathrm{S}$ where the phase is about $50^{\circ} \mathrm{E}$. Hence, the peak of positive temperature perturbation is 
located above the southwest edge of Hellas. This is in good agreement with the $7 \mathrm{~K}$ peak found by Hinson et al. (2003) near $10 \mathrm{~Pa}$ and $60^{\circ} \mathrm{S}$, with a phase of $\sim 40^{\circ} \mathrm{E}$. The differences in the peak amplitude may be due to the fact that we are considering the absolute difference between the peak temperature and the adjacent regions, while Hinson et al. (2003) report the wave amplitude after the zonal average has been subtracted.

All the elements summarized in this section allow us to suggest an explanation for the results in figure 7a: a wavenumber-one planetary wave establishes a high-pressure zone over much of the eastern hemisphere, preventing $\mathrm{CO}_{2}$ condensation in the atmosphere during the fall season. Upward motions occur in the western hemisphere, establishing a cold, low-pressure zone. This makes the $\mathrm{CO}_{2}$ precipitation very likely to occur in the western hemisphere. As explained in section 1.1, upward motions result in an efficient adiabatic cooling, allowing high $\mathrm{CO}_{2}$ condensation rate in the areas where this occurs. The two climates established by the topographically forced circulation produce differences in the nature of the surface ice deposits. Thus topography, through its influence on the circulation, is ultimately responsible for the nature and location of the RSPC. As discussed in section 7, these results confirm and extend what has been recently proposed by Colaprete et al. (2005). 


\section{3 - Comparison with an atmospheric model}

Global circulation models provide a global picture of all atmospheric variables. This can be essential to put the observations in the right context, especially those which are unevenly distributed in space and time.

We have performed a comparison of selected PFS orbits with the results provided by the martian GCM of the Laboratoire de Météorologie Dynamique (LMD) in Paris (Forget et al, 1999), which is an adaptation to Mars of the Terrestrial LMDZ model in use at LMD since the 80's (Sadourny and Laval, 1984, and Hourdin et al., 2006).

Figure 12 shows a profile-to-profile comparison between observations and model results at $L_{S}=52.21$ and $86^{\circ} \mathrm{S}$ in the "cold" hemisphere, where the solid profiles are PFS retrievals at different longitudes in the coldest region (indicated by a blue arrow in figure 7a) and the dot-dashed ones are the corresponding model profiles.

[Fig. 12]

The general agreement among the profiles is very good for most of the atmospheric column where PFS is mostly sensitive. Even the small inversions between 4 and 5 mbar are in good agreement, as well as the more pronounced polar warming inversions higher than 0.1 mbar (although, at that particular altitude, they are not observed by PFS at all longitudes). For the purposes of this paper, the good agreement in the "cold" 
hemisphere shows that $\mathrm{CO}_{2}$ snowfall may indeed occur in a large range of atmospheric altitudes, other than by direct condensation on the ground.

Figure 13 shows the same kind of comparison for the "warm" hemisphere at $L_{S}=52.46$ and $86^{\circ} \mathrm{S}$. This reveals that the $\mathrm{GCM}$ predicts $\mathrm{CO}_{2}$ condensation in the whole atmospheric column from the ground up to $0.1 \mathrm{mbar}$ (dot-dashed profile), whereas the PFS retrievals are much warmer (up to 10 degrees). The altitude of the polar warming inversion is in good agreement, but neither its temperature nor the inversion at the ground is close to the observations. The GCM, in this configuration, is not able to show any asymmetry between the eastern and western hemispheres, predicting $\mathrm{CO}_{2}$ snowfall at all longitudes during the southern polar night.

[Fig. 13]

The effect of having different atmospheric dust loading has been taken into account, but did not change the results significantly. What does change the model results is the use of a different implementation for the integration of the dynamical equations, together with the use of a different filter for the numerical stability around the pole. The dashed curve in Fig. 13 is the GCM profile produced by using a later version of the (finite differences) dynamical core of the LMDZ model (LMDZ-3), whereas the dot-dashed profile is produced with the version LMDZ-2 (used in Forget et al., 1998, and Forget et 
al., 1999). The former lies within 5 degrees from the average of the PFS retrievals at most altitudes, although the polar warming starts at higher altitude than the observations. The GCM based on the new dynamical core is able to take into account warm temperatures in the eastern hemisphere, but fails to predict cold atmospheric temperatures in the western one, thus preventing $\mathrm{CO}_{2}$ snowfall.

Figure 13 shows also that the GCM based on the new dynamical core predicts the strong inversion at the ground in the "warm" hemisphere. It must be mentioned that this particular feature is not very robust in the PFS retrievals, as the sensitivity of the instrument does not extend so close to the ground. Different retrieval methods which make use of different a priori profiles show different results around 4 mbar for the curves in Fig. 13 (not shown here). Nevertheless, at higher altitudes the retrievals are robust and do not depend on the chosen a priori profile, therefore the warm temperatures are consistently observed no matter which retrieval method is used. This supports the evidence that, in the eastern martian hemisphere, $\mathrm{CO}_{2}$ snowfall does not occur in the bulk of the atmosphere, and therefore most $\mathrm{CO}_{2}$ condensation must occur very close to the ground (e.g. direct surface or near-surface condensation).

What can explain the different results obtained with the two versions of the model? The physical parameterizations are the same in both versions, and the difference between the two dynamical cores is very subtle. Although these cores are based on the same derivation of the primitive equations of the meteorology and use the same grid and 
integration time, they differ by the way they were implemented in the model. In particular, they use different sets of dynamical variables, a different vertical discretization and different stability filters at the poles. In simulations of Earth's climate, these two versions of the dynamical core have provided identical results, as they do for the case of Mars outside the polar night regions. During the polar nights, the radiative forcing is reduced to radiative cooling, and our simulations show that the martian atmosphere becomes extremely sensitive to the dynamical forcing. This suggests that the presence of a hemispherical asymmetry as shown by PFS observations is indeed a dynamical effect which is not yet reproduced by the model, although each of the two different versions is able to yield one of the two observed regional climates. When extending the comparison between observations and model at several latitudes, it appears clear that it is at high latitudes (corresponding to the polar night region) where the disagreement is more pronounced, whereas model (both versions) and observations agree very well at all other latitudes

In figure 14 we plot the time-averaged zonal means of temperature for the two versions of the GCM in the range of areocentric longitude $50^{\circ}-70^{\circ}$, comparing with the PFS observations at two local times (7a.m. and 7p.m). The most prominent differences are located around the polar vortex, which exhibits a profoundly different structure in all the panels of the figure. The observations from PFS show that the periphery of the polar vortex is highly barotropic, with little or no entrainment of polar warming at high 
latitudes, at almost all altitudes. The model, on the contrary, predicts that the polar warming breaks the vertical extension of the vortex, locating the position of the warming at much higher latitudes than the observations. This, in turn, would imply quite different properties in the transport of tracers at high latitudes during the polar night. Two remarkable differences between the two versions of the model are the stronger polar warming predicted by the version LMDZ-2 (old dynamical core) at both local times, and the colder temperatures shown in the polar vortex, especially at low altitudes and near the ground (as already described for Figs. 12-13). At latitudes equatorward of $50^{\circ} \mathrm{S}$ the agreement between the two versions of the model and the observations is excellent for pressures higher than $0.1 \mathrm{mb}$, whereas temperatures are underestimated in the model for lower pressures, mainly in the early morning.

[Fig. 14]

Keeping in mind the disagreement between model and observations that we have highlighted in the polar night region, we compare in Figure 15 the temperatures observed by PFS and the corresponding model temperatures during orbit 695, which crosses entirely the south pole, from the "warm" hemisphere $\left(\sim 70^{\circ} \mathrm{E}\right.$ longitude $)$ to the "cold" one $\left(\sim 250^{\circ} \mathrm{E}\right.$ longitude). This orbit (already analysed in section 5.2$)$ is at Ls $=$ 
$70^{\circ}$, thus the temperatures at high latitudes in the polar vortex are colder than those shown in Figs. 13.

The structure of the two hemispheres, as described in Fig. 11, appears remarkably similar in the observations and in the model, apart from the differences related to the structure of the polar vortex. The asymmetry between the longitudes around the Hellas basin and the western longitudes is evident also in the model, independently from the version which is used. Although the model is not able to reproduce the asymmetry between eastern and western longitudes in the polar night (as shown in Figs. 12-13), the agreement with the observations at latitudes outside the polar night supports the hypothesis of a wavenumber-one planetary wave induced by the presence of the underlying topography, which the model is not able to yield inside the polar night region.

Outside the polar region one can also appreciate that the most prominent difference is located around the equator. The underestimation of temperatures at the equator and above $0.2 \mathrm{mb}$ in the two versions of the model might be explained (at this time of the year and at these altitudes) by the presence of water ice clouds in the tropics, an effect which is not taken into account in the simulations (see Wilson et al., 2007, for details on this topic).

[Fig. 15] 


\section{6 - The late-fall and winter polar cap}

At the end of the fall season, the atmosphere above the polar region cools down and evolves toward the situation described in Colaprete et al. (2005) for the winter season. The polar cap reaches its maximum extension and starts to shrink. $\mathrm{CO}_{2}$ condensation in the atmosphere is now allowed at lower latitudes as well as in the eastern hemisphere. As an example, we show in figure 16 the air temperature field difference $\Delta \mathrm{T}_{\text {sat }}(\mathrm{z})=\mathrm{T}(\mathrm{z})$ $-\mathrm{T}_{\mathrm{sat}}(\mathrm{z})$ for orbit $755\left(\mathrm{Ls}=78^{\circ}\right)$. The track of PFS measurements is depicted in the topright panel. White circles highlight the areas where the $\mathrm{CO}_{2}$ condensation is allowed, according to the vertical profiles shown in the bottom panel.

[Fig. 16]

The key observational aspects of the southern hemisphere winter weather have been recently summarized by Barnes and Tyler (2007). During the winter, the atmosphere frequently chills to below the condensation temperature of $\mathrm{CO}_{2}$ and becomes supersaturated. The largest and most persistent supersaturation occurs in a region which extends from the areas southward of the Tharsis plateau as far east as the vicinity of the Argyre basin. More broadly, the western hemisphere exhibits much stronger storm activity than the eastern hemisphere, and is also substantially colder at a given altitude, on average. In other words, $\mathrm{CO}_{2}$ condensation in the atmosphere is now allowed in both 
hemispheres, but the low-pressure zone established by the planetary wave over much of the western hemisphere allows a higher $\mathrm{CO}_{2}$ condensation rate than in the eastern region.

Colaprete et al. (2005) have shown that for $\mathrm{Ls}=90^{\circ}-110^{\circ}$, at $-75^{\circ}$ latitude, the temperature of the whole atmospheric column (up to $\sim 0.3 \mathrm{mb}$ ) is always below or far below the $\mathrm{T}_{\text {sat }}$ profile (up to more than $20 \mathrm{~K}$ in the western hemisphere). With our current dataset described in Section 1.1, for Ls $>90^{\circ}$ we observe latitudes poleward of $70^{\circ}$ with only six orbits, four of which extend below $-85^{\circ} \mathrm{S}$ latitude in the western hemisphere. Although more observations may be needed for a complete discussion, it is interesting to note that our observations show a different situation than that described in Colaprete et al. (2005). In our data, areas of atmospheric condensation are present poleward of $70^{\circ} \mathrm{S}$ latitude, but the atmosphere is far from being completely supersaturated at $75^{\circ} \mathrm{S}$ latitude. A completely supersaturated atmosphere, from the ground up to $\sim 40 \mathrm{~km}$ altitude and at all the longitudes, is observed exclusively at latitudes higher than $80^{\circ} \mathrm{S}$. This is true for each of the four cases examined.

\section{7 - Discussion and Conclusion}

We have analyzed the condensing $\mathrm{CO}_{2}$ south polar cap of Mars during the fall and winter seasons. In the early-fall season $\left(\mathrm{Ls} 0^{\circ}-25^{\circ}\right)$ the $\mathrm{CO}_{2}$ condensation in the 
atmosphere is not allowed, the atmosphere being several degrees warmer than the saturation temperature. The cap extends up to $\sim 70^{\circ} \mathrm{S}$ latitude and consists essentially of $\mathrm{CO}_{2}$ frost deposits (direct vapor deposition). This is likely to be the situation until the middle of the fall season, with the exception that the deposits extend up to $\sim 60^{\circ} \mathrm{S}$ latitude at that time. During the first half of the fall season, the cap edges advance symmetrically with a constant speed of about $10^{\circ}$ of latitude per $15^{\circ} \mathrm{Ls}$, at every longitude. At Ls $=70^{\circ}$ the cap is near to its maximum extension of $\sim 40^{\circ} \mathrm{S}$ latitude. It appears asymmetric, due to the presence of the two major martian basins, Hellas and Argyre, where the $\mathrm{CO}_{2}$ frost can be stable at higher temperatures, thanks to higher surface pressures inside the basins.

In the Ls $50^{\circ}-70^{\circ}$ period, the south pole of Mars is characterized by two distinct regional climates: $\mathrm{CO}_{2}$ snow falls are allowed exclusively in the western hemisphere, where the atmospheric temperatures goes below the condensation temperature of the gaseous $\mathrm{CO}_{2}$ at given altitudes. The longitudinal thermal asymmetries seen between the western and eastern hemispheres in southern high latitudes are the result of a planetary wave that is excited primarily by the middle-latitude surface topography, namely Hellas and Argyre. This wavenumber-one planetary wave establishes a high-pressure zone over much of the eastern hemisphere, preventing $\mathrm{CO}_{2}$ condensation in the atmosphere. Upward motions occur in the western hemisphere, establishing a cold, low-pressure zone. This makes the $\mathrm{CO}_{2}$ precipitation very likely to occur in the western hemisphere. 
The differences in the nature of the surface ice deposits during the mid-late fall season are the main responsible for the residual south polar cap asymmetry. These results confirm and extend what has been recently proposed by Colaprete et al. (2005).

The explanation suggested by Colaprete et al. (2005) for the RSPC asymmetry involves a different $\mathrm{CO}_{2}$ condensation rate in the atmosphere in the two hemispheres above the south polar region during the southern winter, the higher condensation rate occurring in the western hemisphere. PFS observations shown in this paper reveal that an important role is also played by the fall season, expecially during the $50^{\circ}-70^{\circ}$ Ls range. In this period, in fact, $\mathrm{CO}_{2}$ precipitation occurs exclusively in the western hemisphere, and particularly in the longitudinal corridor of the RSPC. Prettyman et al. (2003) have shown that the measured inventory of $\mathrm{CO}_{2}$ frost in the southern seasonal cap achieves a maximum value of $8 \times 10^{18} \mathrm{~g}$ between $160^{\circ}$ and $170^{\circ} \mathrm{Ls}$, which is slightly more than $30 \%$ of the established value for total atmospheric mass $\left(2.5 \times 10^{19} \mathrm{~g}\right)$. They have also shown that, by $\mathrm{Ls}=70^{\circ}$ at $85^{\circ} \mathrm{S}$ latitude, more than a third of such amount of $\mathrm{CO}_{2}$ ice has been already deposited in the polar cap. As a consequence of the results discussed in section 5.1, we can reasonably assume that most of this $\mathrm{CO}_{2}$ ice is deposited within the western hemisphere and within the longitudinal corridor of the RSPC. We note here that all the $\mathrm{CO}_{2}$ ice clouds and precipitations phenomena previously reported (Formisano et al., 2006; Montmessin et al., 2006; Montmessin et al., 2007) form inside supersaturated pockets of air created by upward propagating thermal waves. In fact, upward motions in 
the atmosphere cause the temperature to rapidly decrease to a supersaturation level, allowing $\mathrm{CO}_{2}$ condensation in the atmosphere. Unfortunately, due to the limitations in the retrieval scheme described in section 1.2, we cannot state with the current version of the PFS retrievals whether the atmospheric temperature goes below the saturation point or not; nevertheless, the considerations described above, the model predictions, and the strong upward motions associated with the planetary wave in the colder hemisphere make the condensation and precipitation scenario very likely.

Frost grain size is determined by the style of frost deposition. Smaller grains form when there is atmospheric precipitation to the surface and larger grains form when frost is directly deposited to the surface. Smaller grains result in higher albedo and vice versa (Calvin and Martin, 1994; Warren et al., 1990). Moreover, the higher the albedo, the lower the sunlight absorbed and, consequently, the sublimation rate (see e.g. James et al., 1992, and Thomas et al., 1992). Therefore, the different amounts of $\mathrm{CO}_{2}$ ice deposited in the two hemispheres during the fall and winter seasons, together with the different sublimation rates due to different grain sizes and, in turn, different albedo between $\mathrm{CO}_{2}$ (fresh) snow and frost, may explain the asymmetry observed at the end of the southern summer: the dry ice sublimes entirely in the eastern hemisphere, while in the western hemisphere it survives all year long as the RSPC.

Albedo, deposition type and amount of ice deposited are all related. In the polar night we always have $\mathrm{CO}_{2}$ condensing directly onto the surface. This results from IR cooling; 
but, at a given latitude, the onset of this process is primarily controlled by the amount of heat stored in summer and released in fall/winter (thus by the amount of time without frost and by the seasonal subsurface thermal inertia). The albedo also affects the surface accumulation in the polar night, because keeping frost later in spring reduce the amount of heat stored in the subsurface during summer. An extreme example is the South permanent polar cap where the surface condensation is maximum since there is no subsurface heating. In addition, as we have shown, $\mathrm{CO}_{2}$ can efficiently condense in the atmosphere (precipitation), especially in areas where adiabatic cooling occurs. Precipitation impacts not only the amount of ice deposited (higher in the longitudinal corridor of the RSPC) but also the grain size and thereby the cap albedo, which plays a key role outside the polar night. The ice accumulation is longitude-dependent, and so are the albedo and the south polar cap sublimation. The albedo increase with time is longitude-dependent too (Schmidt et al., 2007). In conclusion, the albedo (with the different related processes, such as dust contamination, ice metamorphism, deposition type) and the different amounts of $\mathrm{CO}_{2}$ ice deposited in the two hemispheres are the key factors that control the south polar cap recession.

In this paper we have also performed a comparison of selected PFS orbits with the results provided by the martian GCM of the Laboratoire de Météorologie Dynamique in Paris in the range of areocentric longitude $50^{\circ}-70^{\circ}$. The aims of this comparison were 
1) 1) to confirm and support the observations, putting them in the global context of the simulated martian atmospheric dynamics, and

2) to provide a validation of the results of the GCM in the polar night, where the model has shown to be very sensitive to the numerical implementation of the equations of the dynamics.

The results of the comparison show that, at latitudes outside the polar night region, model and observations agree remarkably well. In the polar night region, the position and strength of the polar warming, together with the temperatures at the base of the polar vortex, are in disagreement between the two versions of the model used in the present work, and in partial disagreement with the observations (roughly, the model based on the LMDZ-2 dynamical core disagrees with the observations in the eastern hemisphere whereas the version based on the LMDZ-3 dynamical core disagrees in the western hemisphere). The comparison included in this paper confirms that modeling the circumpolar region during the period of the polar night is a crucial task for the GCMs.

Work is in progress to understand the role played by different dynamical cores (including a spectral dynamical core which is part of the UK MGCM) and numerical stability filters in modelling the climate of the southern polar night.

Future work will be devoted to extend this first cross-validation of MEX/PFS and martian GCMs to other seasons and latitudes, and to investigate in more detail the atmospheric dynamics and the transport of tracers in the polar night regions, where PFS 
observations (together with future observations from NASA's Mars Reconnaissance Orbiter/Mars Climate Sounder) are essential.

\section{Acknowledgments}

The PFS experiment has been built at the Istituto di Fisica dello Spazio Interplanetario (IFSI) of Istituto Nazionale di Astrofisica (INAF), and has been founded by the Italian Space Agency (ASI) in the context of the Italian participation to the Mars Express mission of ESA.

The authors wish to thank the reviewers (P. James and T. N. Titus) for their constructive comments and suggestions. 


\section{References}

Andrews, D. G., J. R. Holton, and C. B. Leovy, 1987. Middle Atmosphere Dynamics, Academic, San Diego, Calif.

Banfield, D., Conrath, B. J., Smith, M. D., Christensen, P. R., and John Wilson, R., 2003. Forced waves in the martian atmosphere from MGS TES nadir data. Icarus, 161, 319-345.

Banfield, D., Conrath, B. J., Gierasch, P. J., Wilson, R. John, Smith, M. D., 2004. Traveling waves in the martian atmosphere from MGS TES Nadir data. Icarus, 170, $365-403$.

Barnes, J. R., 1980. Time Spectral Analysis of Midlatitude Disturbances in the martian Atmosphere. J. Atmos. Sci., 37, 2002-2015.

Barnes, J. R., 1981. Midlatitude Disturbances in the martian Atmosphere: A Second Mars Year. J. Atmos. Sci., 38, 225-234.

Barnes, J. R., J. Pollack, R. Haberle, C. Leovy, R. Zurek, H. Lee, and J. Schaeffer, 1993. Mars atmospheric dynamics as simulated by the NASA Ames generalcirculation model. 2. Transient baroclinic eddies. J. Geophys. Res., 98, 3125-3148. Barnes, J. R., 2003. Mars Weather Systems and Maps: FFSM Analyses of MGS TES Temperature Data. The Sixth International Conference on Mars, Pasadena, California, 20 - 25 July, abstract no.3127. 
Barnes, J. R. and D. Tyler, 2007. Winter weather on Mars: the unique southern hemisphere. The Seventh International Conference on Mars, Pasadena, California, 9 - 13 July, abstract no.3094.

Bibring, J-P., Langevin, Y., Poulet, F., Gendrin, A., Gondet, B., Berthé, M., Soufflot, A., Drossart, P., Combes, M., Bellucci, G., Moroz, V., Mangold, N., Schmitt, B. and the OMEGA team, 2004. Perennial water ice identified in the south polar cap of Mars. Nature, 428, 627-630.

Calvin, W.M., Martin, T.Z., 1994. Spatial variability in the seasonal south polar cap of Mars. J. Geophys. Res., 99, 21143-21152.

Colaprete, A., and O. B. Toon, 2002. Carbon dioxide snow storms during the polar night on Mars, J. Geophys. Res., 107, 5051.

Colaprete, A., Barnes, J. R., Haberle, R. M., Hollingsworth, J. L., Kieffer, H. H., Titus, T. N., 2005. Albedo of the south pole of Mars determined by topographic forcing of atmosphere dynamics. Nature, 435, 184-188.

Conrath, B. J., J. C. Pearl, M. D. Smith, W. C. Maguire, P. R. Christensen, S. Dason, and M. S. Kaelberer, 2000. Mars Global Surveyor Thermal Emission Spectrometer (TES) observations: Atmospheric temperatures during aerobraking and science phasing. J. Geophys. Res., 105, 9509-9519.

Douté, S., B. Schmitt, Bibring, J-P., Langevin, Y., Altieri, F., Bellucci, G., Gondet, B. and the MEX OMEGA Team, 2005. Nature and Composition of the Icy Terrains of 
the South Pole of Mars from MEX OMEGA Observations. Lunar and Planetary Science XXXVI.

Eluszkiewicz, J., On the microphysical state of the martian seasonal caps, Icarus, 103, 43-48, 1993.

Forget, F., J. B. Pollack, and G. B. Hansen, 1995. Low brightness temperatures of martian polar caps: $\mathrm{CO}_{2}$ clouds or low surface emissivity?. J. Geophys. Res., 100, $21,119-21,234$.

Forget, F., Hourdin, F., and Talagrand, O., 1998. $\mathrm{CO}_{2}$ snowfall on Mars: Simulation with a General Circulation Model. Icarus, 131, 302-316.

Forget, F., F. Hourdin, F. Fournier, C. Hourdin, O. Talagrand, M. Collins, S. R. Lewis, P. L. Read, and J.-P. Huot, 1999. Improved general circulation models of the martian atmosphere from the surface to above $80 \mathrm{~km}$. J. Geophys. Res., 104, $24,155-24,176$.

Forget, F., E. Millour, S. Lebonnois, L. Montabone, K. Dassas S. R. Lewis, P. L. Read, M. Lopez-Valverde, F. Gon-zalez Galindo F. Montmessin, F. Lefevre M.C. Desjean and J. P. Huot, 2006. The New Mars Climate Database, in 2nd workshop on Mars atmosphere modelling and observations abstract book, Granada, Spain.

Formisano, V., and the PFS Team, 2005. The Planetary Fourier Spectrometer (PFS) onboard the European Mars Express mission. Planet. Space Sci., Volume 53, issue 10, 963-974. 
Formisano, V., Maturilli, A., Giuranna, M., D’Aversa, E., Lopez-Valverde, M. A., 2006. Observations of non-LTE emission at 4.5 microns with the planetary Fourier spectrometer abord the Mars Express mission. Icarus, 182, 51-67.

Gierasch, P. J., and R. M. Goody, 1968. A study of the thermal and dynamical structure of the martian lower atmosphere. Planet. Space Sci. 16, 615-646.

Giuranna, M., Formisano, V., Biondi, D., Ekonomov, A., Fonti, S., Grassi, D., Hirsch, H., Khatuntsev, I., Ignatiev, N., Michalska, M., Mattana, A., Maturilli, A., Moshkin, B. E., Mencarelli, E., Nespoli, F., Orfei, R., Orleanski, P., Piccioni, G., Rataj, M., Saggin, B., Zasova, L., 2005a. Calibration of the Planetary Fourier Spectrometer short wavelength channel. Planet. Space Sci., Volume 53, issue 10, 975-991.

Giuranna, M., Formisano, V., Biondi, D., Ekonomov, A., Fonti, S., Grassi, D., Hirsch, H., Khatuntsev, I., Ignatiev, N., Malgoska, M., Mattana, A., Maturilli, A., Mencarelli, E., Nespoli, F., Orfei, R., Orleanski, P., Piccioni, G., Rataj, M., Saggin, B., Zasova L., 2005b. Calibration of the Planetary Fourier Spectrometer long wavelength channel. Planet. Space Sci., Volume 53, issue 10, 993-1007.

Giuranna, M., V. Formisano, D. Grassi and A. Maturilli, 2007a. Tracking the edge of the south seasonal polar cap of Mars. Planet. Space Sci., Volume 55, Issue 10, $1319-1327$. 
Giuranna, M., Hansen, G., Maturilli, A., Zasova, L., Formisano, V., Grassi, D., Ignatiev, N., 2007b. Spatial variability, composition and thickness of the seasonal north polar cap of Mars in mid-spring. Planet. Space Sci., Volume 55, Issue 10, 1328-1345.

Grassi, D., Ignatiev, N. I., Zasova, L. V., Maturilli, A., Formisano, V., Bianchini, G. A., Giuranna, M., 2005. Methods for the analysis of data from the Planetary Fourier Spectrometer on the Mars Express Mission. Planet. Space Sci., Volume 53, Issue 10, 1017-1034.

Grassi D., F. Forget, C. Fiorenza, N.I. Ignatiev, A. maturilli, L.V. Zasova, V. Formisano, 2007. The martian Atmosphere in the Region of Hellas Basin as Observed by the Planetary Fourier Spectrometer (PFS-MeX). Planet. Space Sci., doi:10.1016/j.pss.2006.12.006.

Hansen, G. B., Giuranna, M., Formisano, V., Fonti, S., Grassi, D., Hirsh, H., Ignatiev, N., Maturilli, A., Orleanski, P., Piccioni, G., Rataj, M., Saggin, B., Zasova, L., 2005. PFS-MEX observation of ices in the residual south polar cap of Mars. Planet. Space Sci., 53, issue 10, 1089-1095.

Hess, S. L., Ryan, J. A., Tillman, J. E., Henry, R. M., Leovy, C. B., 1980. The annual cycle of pressure on Mars measured by Viking landers 1 and 2. Geoph. Res. Lett., 7, 197-200.

Hinson, D. P. and R.J. Wilson, 2002. Transient waves in the southern hemisphere of Mars. Geophys. Res. Lett., 29(7), doi: 10.1029/2001GL014103. 
Hinson, D. P., R. J. Wilson, M. D. Smith, and B. J. Conrath, 2003. Stationary planetary waves in the atmosphere of Mars during southern winter. J. Geophys. Res., 108, E1, 5004.

Hollingsworth, J. L., and J. R. Barnes, 1995. Forced Stationary Planetary Waves in Mars's Winter Atmosphere. J. Atmos. Sci., 53, 428-448.

Holton, J. R., 1979, An introduction to dynamic Meteorology, Academic, San Diego, Calif.

Hourdin, F., P. Le Van, F. Forget, and O. Talagrand, 1993. Meteorological variability and the annual surface pressure cycle on Mars. J. Atmos. Sci., 50, 3625-3640.

Hourdin, F., F. Forget, and O. Talagrand, 1995. The sensitivity of the martian surface pressure to various parameters: A comparison between numerical simulations and Viking observations. J. Geophys. Res., 100, 5501-5523.

Hourdin, F., I. Musat, S. Bony, P. Braconnot, F. Codron, J.-L. Dufresne, L. Fairhead, M.-A. Filiberti, P. Friedlingstein, J.-Y. Grandpeix, G. Krinner, P. Levan, Z.-X. Li, F. Lott, 2006. The LMDZ4 general circulation model: climate performance and sensitivity to parametrized physics with emphasis on tropical convection, Climate Dynamics, 27, 787-813.

Ivanov, A. B., D. O. Muhleman, 2001. Cloud Reflection Observations: Results from the Mars Orbiter Laser Altimeter. Icarus, 154, 190-206. 
Jakosky, B. M., and R. M. Haberle, 1990. Year-to-year instability of the Mars south polar cap. J. Geophys. Res., 95, 1359-1365.

James, P. B., H. H. Kieffer, D. A. Paige, in Mars, H. H. Kieffer et al., Eds. (Univ. of Arizona Press, Tucson, AZ, 1992), pp. 934-968.

Kieffer, H. H., S. C. Chase, E. D. Miner, F. D. Palluconi, G. Münch, G. Neugebauer, and T. Z. Martin, 1976. Infrared thermal mapping of the martian surface and atmosphere: First results. Science, 193, 780-786.

Kieffer, H. H., 1979. Mars south polar spring and summer temperatures: a residual $\mathrm{CO}_{2}$ frost. J. Geophys. Res., 84, 8263-8288.

Kieffer, H. H., 1990. $\mathrm{H}_{2} \mathrm{O}$ grain size and the amount of dust in Mars's residual north polar cap. J. Geophys. Res., 95, 1481-1493.

Kieffer, H. H., Titus, T. N., and Mullins, K.F., 2000. Mars south polar spring and summer behavior observed by TES: Seasonal cap evolution controlled by frost grain size. J. Geophys. Res., 105, 9653-9699.

Kieffer, H. H., and Titus, T. N., 2001. TES Mapping of Mars' North Seasonal Cap. Icarus, 154, 162-180.

Leovy, C., 1969. Theoretical aspects of meteorology. Appl. Opt., Vol. 8, No. 7 , 12791286.

Leovy, C., 2001. Weather and climate on Mars. Nature, 412, 245-249. 
Montmessin, F., Bertaux, J. L., Quemerais, E., Korablev, O., Rannou, P. , Forget, F., Perrier, S., Fussen, D. , Lebonnois, S, Reberac, A., Dimarellis, E., 2006. Subvisible $\mathrm{CO}_{2}$ ice clouds detected in the mesosphere of Mars. Icarus, vol. 183, p. 403.

Montmessin, F., B. Gondet, J.P. Bibring, T. Fouchet, F. Forget, P. Drossart, Y. Langevin, T. Encrenaz, 2007. Hyperspectral imaging of $\mathrm{CO}_{2}$ ice clouds on Mars. EGU General assembly 2007, Vienna, Austria, 15 - 20 April.

Paige, D. A., D. Crisp, and M. L. Santee, 1990. It snows on Mars. Bull. Am. Astron. Soc., 22, 1075.

Paige, D. A., and Ingersoll, 1985. Annual heat balance of martian polar caps: Viking observations. Science, 228, 1160-1168.

Pollack, J. B., R. M. Haberle, J. Schaeffer, and H. Lee, 1990. Simulations of the general circulation of the martian atmosphere. 1. Polar processes. J. Geophys. Res. 95, $1447-1473$.

Prettyman, T. H., Feldman, W. C., Murphy, J. R., Funsten, H. O., Lawrence, D. J., Linn, R. R., Maurice, S., Tokar, R. L., 2003. Seasonal Advance and Retreat of Mars' South Polar Cap as Measured by the Mars Odyssey Neutron Spectrometer. Third International Conference on Mars Polar Science and Exploration. October 13 - 17, Alberta, Canada, abstract no.8099.

Pettengill, G. H. and Ford, P. G., 2000. Winter Clouds over the North martian Polar Cap. Geoph. Res. Lett., 27, 609-612. 
Sadourny, R. and K. Laval, 1984. January and July performance of the LMD general circulation model. In "New Perspectives in Climate Modeling", A.L. Berger and C. Nicolis (Eds.), Elsevier Press, Amsterdam, 173-197.

Schmidt, F., S. Douté, B. Schmitt, Y. Langevin, J.-P. Bibring and the OMEGA Team, 2007. Effect of albedo and surface roughness on the seasonal south cap recession of Mars. Abstract. EMSEC 2007, ESTEC, Noordwijk, The Netherlands, 12 - 16 November, Abstract book p. 116.

Smith, D., and Pearl, J. C., 2001. Thermal Emission Spectrometer results: Mars atmospheric thermal structure and aerosol distribution. J. Geophys. Res., 106, $23,929-23,945$.

Stamnes, K., Tsay, S. C., Wiscombe, W., and Jataweera, K., 1988. Numerically stable algorithm for discrete-ordinate-method radiative transfer in multiple scattering and emitting layered media. Appl. Opt., 27, 2502-2509.

Thomas, P. C., S. W. Squyres, K. E. Herkenhoff, A. Howard, B. C. Murray, in Mars, H. H. Kiefferet al., Eds. (Univ. of Arizona Press, Tucson, AZ, 1992), pp. 767-795.

Thorsteinsson, Th, 2000. Estimating the evolution of crystal size in the north polar ice cap on Mars. Mars Polar Science, abstract.

Titus, T., H. Kieffer, K. F. Mullins, P. R. Christensen, Slab Ice and Snow Flurries in the martian Polar Night, 2001. J. Geophys. Res. 106, 23,181. 
Titus, T.N., 2005. Mars Polar Cap Edges Tracked over 3 Full Mars Years, $36^{\text {th }}$ Annual Lunar and Planetary Science Conference, March 14-18, 2005, in League City, Texas, abstract no.1993.

Tobie, G., F. Forget and F. Lott, 2003. Numerical simulation of the winter polar wave clouds observed by Mars Global Surveyor Mars Orbiter Laser Altimeter. Icarus, 64, $33-49$.

Warren, S.G., Wiscombe, W.J., Firestone, J.F., 1990. Spectral albedo and emissivity of CO2 in martian polar caps: model results. J. Geophys. Res., 95, 14717-14741.

Zuber, M. T., and Smith, D. E., 2003. Observations of the seasonal polar icecaps of Mars at 1064 nm. Third Mars Polar Science Conference, abstract no.8032.

Zurek, R., et al., Dynamics of the atmosphere of Mars, 1992. In "Mars", eds Kieffer et al., 853-933.

Wilson, R. J., S. R. Lewis, L. Montabone and M. D. Smith, 2007. The Influence of Water Ice Clouds on martian Tropical Atmospheric Temperatures, Icarus, submitted. 


\section{Figure Captions (Print Version)}

Figure 1. Typical quality of PFS spectra modeling for different thermal conditions of the atmosphere. Black curves: single spectra measured by PFS. Grey curves: synthetic spectra computed by the algorithm for the scientific analysis of individual calibrated PFS measurements, developed by Grassi et al., 2005. Black arrows indicate the $\mathrm{CO}_{2} \mathrm{Q}-$ branches.

Figure 2. (a) Mosaic of Mars Express OMEGA images showing the residual south polar cap $\left(\operatorname{Ls} 330^{\circ}-360^{\circ}\right)$. It appears clearly asymmetric, the cap center being displaced by $3^{\circ}$ far from the geographic pole. (b) An example of PFS-data fit used to retrieve the RSPC composition. Black: PFS SWC spectrum of the RSPC $\left(86^{\circ} \mathrm{S}, 20^{\circ} \mathrm{W} ; \mathrm{Ls}=338^{\circ}\right)$. Grey: Bi-Directional Reflectance model (DISORT, Stamnes et. al., 1988). Intimate granular mixture of $7-\mathrm{mm} \mathrm{CO}_{2}$ ice, $0.005 \mathrm{wt} \%$ water ice and $0.02 \mathrm{wt} \%$ dust (modified from Hansen et al., 2005).

Figure 3. Surface temperature maps of the South polar region for five Ls ranges covering the fall and winter seasons.

Figure 4. Orbit \# 287 (Ls $\left.17^{\circ}\right)$, a typical early-fall south pole scenario: the $\mathrm{CO}_{2}$ ice deposits consist of $\mathrm{CO}_{2}$ frost directly condensed on the surface. Atmospheric $\mathrm{CO}_{2}$ condensation occurs only occasionally. Top-left: latitude versus time (in seconds) elapsed from the beginning of the acquisition of the first spectrum. This plot can be used to obtain the latitudes in the other figures. Top-right: the surface temperatures (black curve) are "frozen" to the $\mathrm{CO}_{2}$ condensation temperature (140-147 K, depending on the surface altitude - i.e. surface pressure - given by the red curve) up to $-70^{\circ}$ lat, where they finally increase roughly following the sun elevation gradient (light blue). Middle-left: $\mathrm{CO}_{2}$ condensation temperature at different altitudes. Middle-right: the polar crossing of orbit \# 287; white spots indicate when and where $\mathrm{CO}_{2}$ snow fall is allowed, according to the vertical profile of $\mathrm{T}-\mathrm{T}_{\text {sat }}$ shown in the bottom figure. The black area is where the atmospheric temperature, at a given altitude, goes below the $\mathrm{CO}_{2}$ condensation temperature at the same altitude.

Figure 5. Ls $0^{\circ}-25^{\circ}$. Longitude cross-section at $-85^{\circ}$ latitude showing $\mathrm{T}-\mathrm{T}_{\text {sat }}$ as a function of altitude. Red color is used for $\mathrm{T}-\mathrm{T}_{\text {sat }} \geq 20 \mathrm{~K}$. 
Figure 6. Latitude-Ls plot of the south cap edge. Red triangles are measurements inside the Hellas and Argyre basins, where the $\mathrm{CO}_{2}$ frost is stable at lower latitudes due to higher values of surface pressure. The green triangles belong to the narrow range of longitudes $\left[25^{\circ} \mathrm{W}-25^{\circ} \mathrm{E}\right]$, which is in the same hemisphere containing Hellas and Argyre, but outside the basins. Solid lines represent the south polar cap edges measured by TES during MYs 24-25 and 25-26 (Titus, 2005). Black curve is for $180^{\circ} \mathrm{E}$ longitude and red curve is for $70^{\circ} \mathrm{E}$ longitude, that is inside Hellas basin.

Figure 7. (a) Ls $50^{\circ}-55^{\circ}$. Longitude cross-section at $-85^{\circ}$ latitude showing the vertical profile of $\mathrm{T}-\mathrm{T}_{\mathrm{sat}}$. At these Ls the wavenumber-one planetary wave is already well defined and so are the two distinct climates on either side of the pole. (b) $\mathrm{CO}_{2}$ snowfalls are allowed exclusively in the western hemisphere. The coldest region (blue arrow in figure 7a) is located right around the center of the south residual cap (blue dotted line).

Figure 8. PFS retrievals of air temperature profiles at $85^{\circ} \mathrm{S}$ latitude in the $50^{\circ}-70^{\circ} \mathrm{Ls}$ range in the "cold" hemisphere (solid curves) and in the "warm" hemisphere (dashed curves). Dash-dotted curve is the $\mathrm{CO}_{2}$ condensation profile (see text for more details). The dash-dotted curve is the $\mathrm{CO}_{2}$ condensation profile for the average atmospheric condition of the areas where $\mathrm{CO}_{2}$ condensation in the atmosphere is allowed

Figure 9. a. Zonal-mean thermal structure of martian atmosphere in the $50^{\circ}-70^{\circ} \mathrm{Ls}$ range, from the surface up to $50 \mathrm{~km}$. $b$. Zonal-mean component of the zonal wind derived with Eq. (1) for the same range of solar longitudes as in Fig. 8a.

Figure 10. Effects of the wavenumber-one planetary wave on the atmospheric temperature of the polar region at an altitude of $40 \mathrm{~km}$. The situation is similar down to $\sim 15 \mathrm{~km}$. Below $15 \mathrm{~km}$ wavenumber-two becomes significant.

Figure 11. Thermal field along orbit $695\left(\mathrm{Ls}=70^{\circ}\right)$. The temperature asymmetry between the two hemispheres is clearly visible. The unit of the $\mathrm{x}$-axis is seconds elapsed from the beginning of the acquisition of the first spectrum.

Figure 12. Profile-to-profile comparison between observations at different longitudes (solid curves) and corresponding model results (dot-dashed curves) in the "cold" hemisphere. Latitude is $86^{\circ} \mathrm{S}$ and $\mathrm{Ls}=52.21^{\circ}$ (see text for more details). 
Figure 13. Profile-to-profile comparison between observations at different longitudes (solid curves) and corresponding model results using the LMDZ-3 (new) dynamical core (dashed curve) and the LMDZ-2 (old) one (dot-dashed curve) in the "warm" hemisphere. Latitude is $86^{\circ} \mathrm{S}$ and $\mathrm{Ls}=52.46^{\circ}$ (see text for more details).

Figure 14. Comparison between zonal-mean temperature fields retrieved by PFS and predicted by the two versions of the LMD martian GCM, in the $50^{\circ}-70^{\circ}$ Ls range and for two different local times: 7:30 p.m. (left panels) and 7:30 a.m. (right panels).

Figure 15. Comparison between PFS vertical profiles retrieved for orbit 695, and temperature fields provided by the two versions of the GCM for the same geometries and conditions as the observations. The orbit has been divided in two parts, one in the warm hemisphere (left panels) and one in the cold one (right panels).

Figure 16. Orbit \# 755 (Ls $78^{\circ}$ ), a typical late-fall south pole scenario (see text for details). Top-left: latitude versus time (in seconds) elapsed from the beginning of the acquisition of the first spectrum. This plot can be used to obtain the latitudes in the bottom panel. Top-right: track of PFS observations; white spots indicate when and where $\mathrm{CO}_{2}$ snow fall is allowed, according to the vertical profile of $\mathrm{T}-\mathrm{T}_{\text {sat }}$ shown on the bottom panel. Black areas indicate where the atmospheric temperature, at a given altitude, goes below the $\mathrm{CO}_{2}$ condensation temperature for the same altitude. 


\section{Figure Captions (Electronic Version)}

Figure 1. Typical quality of PFS spectra modeling for different thermal conditions of the atmosphere. Black curves: single spectra measured by PFS. Colored curves: synthetic spectra computed by the algorithm for the scientific analysis of individual calibrated PFS measurements, developed by Grassi et al., 2005. Black arrows indicate the $\mathrm{CO}_{2}$ Q-branches.

Figure 2. a) Mosaic of Mars Express OMEGA images showing the residual south polar cap $\left(\operatorname{Ls~} 330^{\circ}-360^{\circ}\right)$. It appears clearly asymmetric, the cap center being displaced by $3^{\circ}$ far from the geographic pole. b) An example of PFS-data fit used to retrieve the RSPC composition. Black: PFS SWC spectrum of the RSPC $\left(86^{\circ} \mathrm{S}, 20^{\circ} \mathrm{W} ; \mathrm{Ls}=338^{\circ}\right)$. Red: BiDirectional Reflectance model (DISORT, Stamnes et. al., 1988). Intimate granular mixture of $7-\mathrm{mm} \mathrm{CO}_{2}$ ice, $0.005 \mathrm{wt} \%$ water ice and $0.02 \mathrm{wt} \%$ dust (modified from Hansen et al., 2005).

Figure 3. Surface temperature maps of the South polar region for five Ls ranges covering the fall and winter seasons.

Figure 4. Orbit \# 287 (Ls $17^{\circ}$ ), a typical early-fall south pole scenario: the $\mathrm{CO}_{2}$ ice deposits consist of $\mathrm{CO}_{2}$ frost directly condensed on the surface. Atmospheric $\mathrm{CO}_{2}$ condensation occurs only occasionally. Top-left: latitude versus time (in seconds) elapsed from the beginning of the acquisition of the first spectrum. This plot can be used to obtain the latitudes in the other figures. Top-right: the surface temperatures (black curve) are "frozen" to the $\mathrm{CO}_{2}$ condensation temperature (140-147 K, depending on the surface altitude - i.e. surface pressure - given by the red curve) up to $-70^{\circ}$ lat, where they finally increase roughly following the sun elevation gradient (light blue). Middle-left: $\mathrm{CO}_{2}$ condensation temperature at different altitudes. Middle-right: the polar crossing of orbit \# 287; white spots indicate when and where $\mathrm{CO}_{2}$ snow fall is allowed, according to the vertical profile of $\mathrm{T}-\mathrm{T}_{\text {sat }}$ shown in the bottom figure. The black area is where the atmospheric temperature, at a given altitude, goes below the $\mathrm{CO}_{2}$ condensation temperature at the same altitude.

Figure 5. Ls $0^{\circ}-25^{\circ}$. Longitude cross-section at $-85^{\circ}$ latitude showing $\mathrm{T}-\mathrm{T}_{\text {sat }}$ as a function of altitude. Red color is used for $\mathrm{T}-\mathrm{T}_{\text {sat }} \geq 20 \mathrm{~K}$. 
Figure 6. Latitude-Ls plot of the south cap edge. Red triangles are measurements inside the Hellas and Argyre basins, where the $\mathrm{CO}_{2}$ frost is stable at lower latitudes due to higher values of surface pressure. The green triangles belong to the narrow range of longitudes $\left[25^{\circ} \mathrm{W}-25^{\circ} \mathrm{E}\right]$, which is in the same hemisphere containing Hellas and Argyre, but outside the basins. Solid lines represent the south polar cap edges measured by TES during MYs 24-25 and 25-26 (Titus, 2005). Black curve is for $180^{\circ} \mathrm{E}$ longitude and red curve is for $70^{\circ} \mathrm{E}$ longitude, that is inside Hellas basin.

Figure 7. (a) Ls $50^{\circ}-55^{\circ}$. Longitude cross-section at $-85^{\circ}$ latitude showing the vertical profile of $\mathrm{T}-\mathrm{T}_{\mathrm{sat}}$. At these Ls the wavenumber-one planetary wave is already well defined and so are the two distinct climates on either side of the pole. (b) $\mathrm{CO}_{2}$ snowfalls are allowed exclusively in the western hemisphere. The coldest region (blue arrow in figure 7a) is located right around the center of the south residual cap (blue dotted line).

Figure 8. PFS retrievals of air temperature profiles at $85^{\circ} \mathrm{S}$ latitude in the $50^{\circ}-70^{\circ} \mathrm{Ls}$ range in the "cold" hemisphere (solid curves) and in the "warm" hemisphere (dashed curves). Dash-dotted curve is the $\mathrm{CO}_{2}$ condensation profile (see text for more details). The dash-dotted curve is the $\mathrm{CO}_{2}$ condensation profile for the average atmospheric condition of the areas where $\mathrm{CO}_{2}$ condensation in the atmosphere is allowed

Figure 9. a. Zonal-mean thermal structure of Martian atmosphere in the $50^{\circ}-70^{\circ} \mathrm{Ls}$ range, from the surface up to $50 \mathrm{~km}$. $b$. Zonal-mean component of the zonal wind derived with Eq. (1) for the same range of solar longitudes as in Fig. 8a.

Figure 10. Effects of the wavenumber-one planetary wave on the atmospheric temperature of the polar region at an altitude of $40 \mathrm{~km}$. The situation is similar down to $\sim 15 \mathrm{~km}$. Below $15 \mathrm{~km}$ wavenumber-two becomes significant.

Figure 11. Thermal field along orbit $695\left(\mathrm{Ls}=70^{\circ}\right)$. The temperature asymmetry between the two hemispheres is clearly visible. The unit of the $\mathrm{x}$-axis is seconds elapsed from the beginning of the acquisition of the first spectrum.

Figure 12. Profile-to-profile comparison between observations at different longitudes (solid curves) and corresponding model results (dot-dashed curves) in the "cold" hemisphere. Latitude is $86^{\circ} \mathrm{S}$ and $\mathrm{Ls}=52.21^{\circ}$ (see text for more details). 
Figure 13. Profile-to-profile comparison between observations at different longitudes (solid curves) and corresponding model results using the LMDZ-3 (new) dynamical core (dashed curve) and the LMDZ-2 (old) one (dot-dashed curve) in the "warm" hemisphere. Latitude is $86^{\circ} \mathrm{S}$ and $\mathrm{Ls}=52.46^{\circ}$ (see text for more details).

Figure 14. Comparison between zonal-mean temperature fields retrieved by PFS and predicted by the two versions of the LMD Martian GCM, in the $50^{\circ}-70^{\circ}$ Ls range and for two different local times: 7:30 p.m. (left panels) and 7:30 a.m. (right panels).

Figure 15. Comparison between PFS vertical profiles retrieved for orbit 695, and temperature fields provided by the two versions of the GCM for the same geometries and conditions as the observations. The orbit has been divided in two parts, one in the warm hemisphere (left panels) and one in the cold one (right panels).

Figure 16. Orbit \# 755 (Ls $78^{\circ}$ ), a typical late-fall south pole scenario (see text for details). Top-left: latitude versus time (in seconds) elapsed from the beginning of the acquisition of the first spectrum. This plot can be used to obtain the latitudes in the bottom panel. Top-right: track of PFS observations; white spots indicate when and where $\mathrm{CO}_{2}$ snow fall is allowed, according to the vertical profile of $\mathrm{T}-\mathrm{T}_{\text {sat }}$ shown on the bottom panel. Black areas indicate where the atmospheric temperature, at a given altitude, goes below the $\mathrm{CO}_{2}$ condensation temperature for the same altitude. 


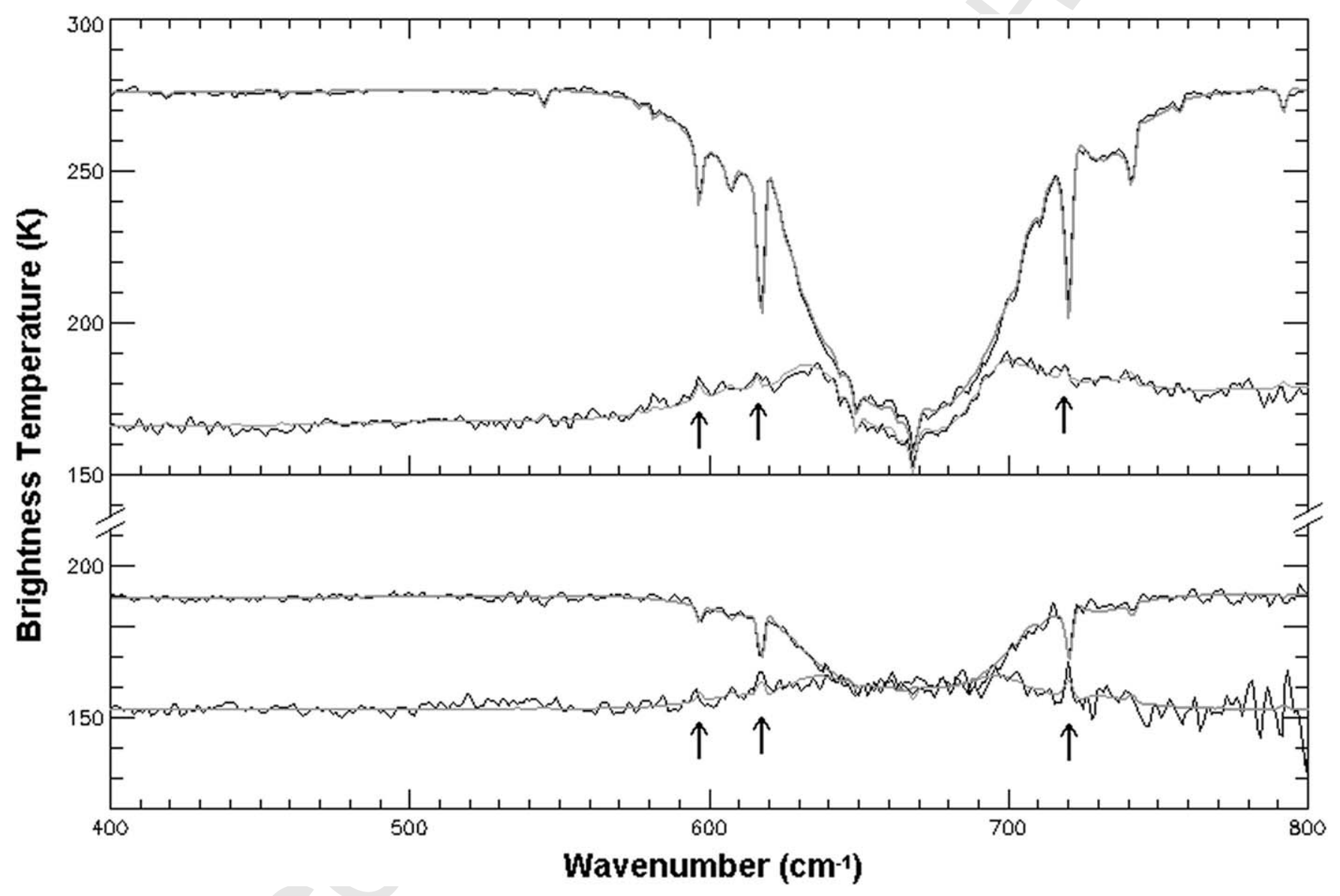




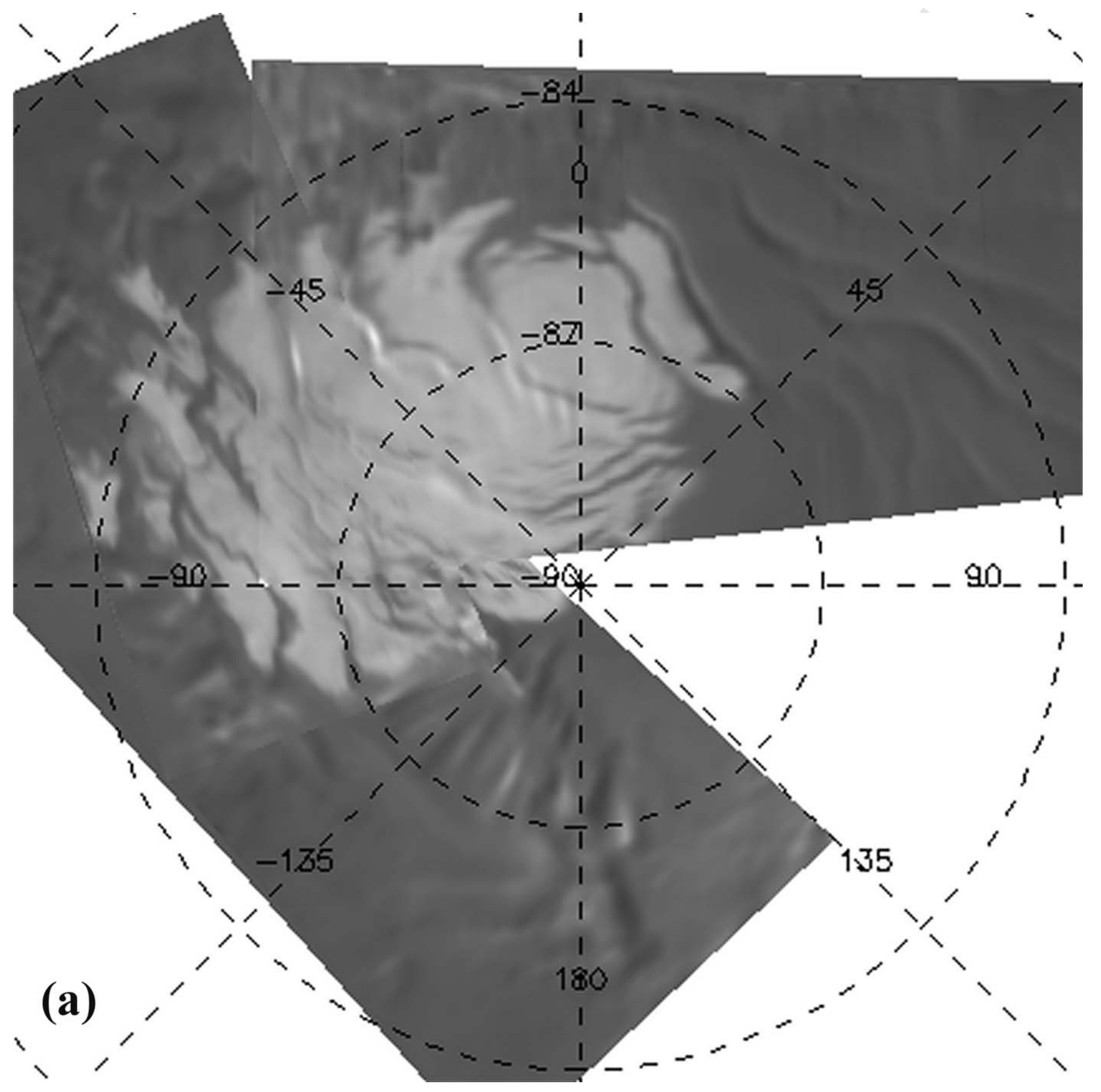




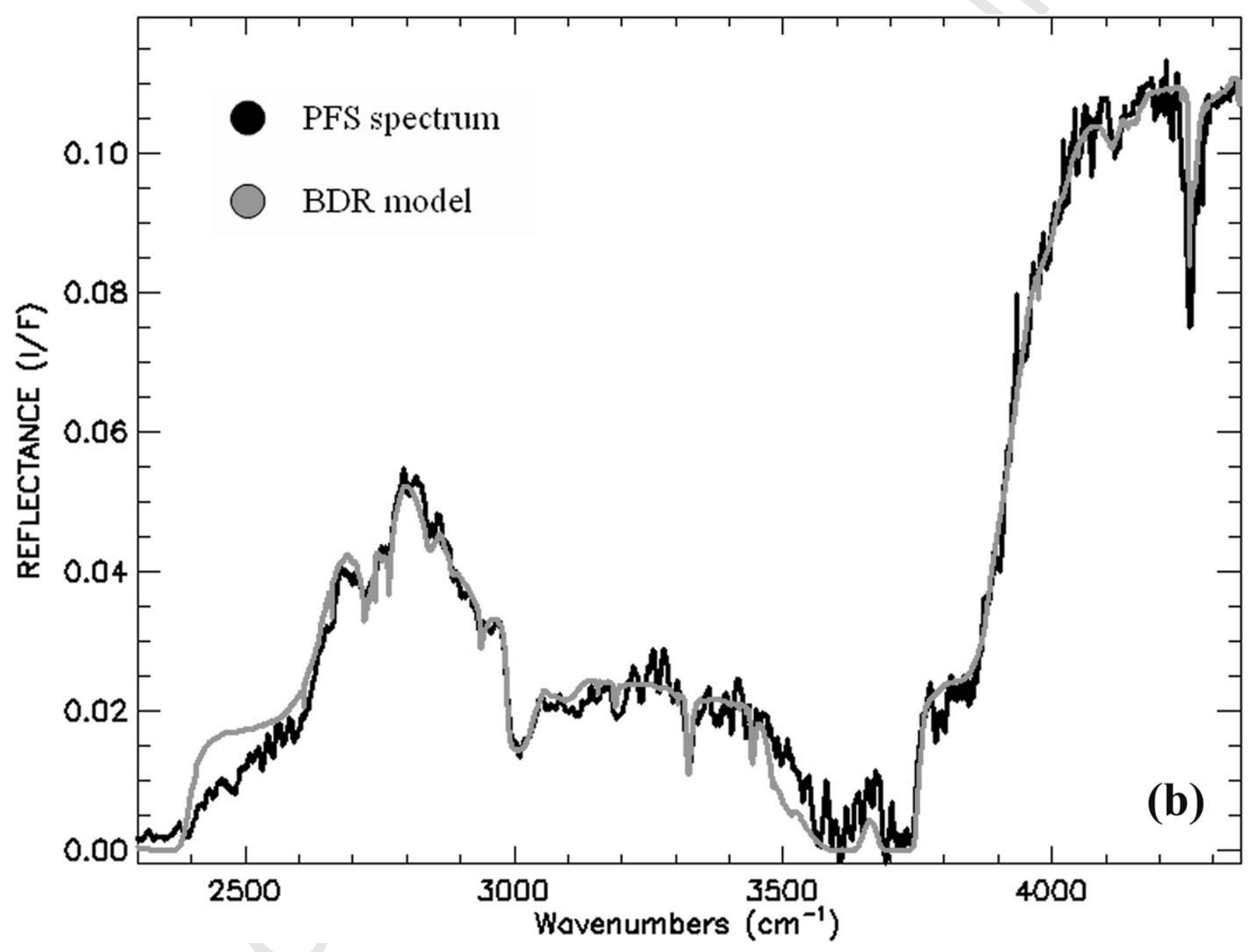




\section{ACCEPTED MANUSCRIPT}
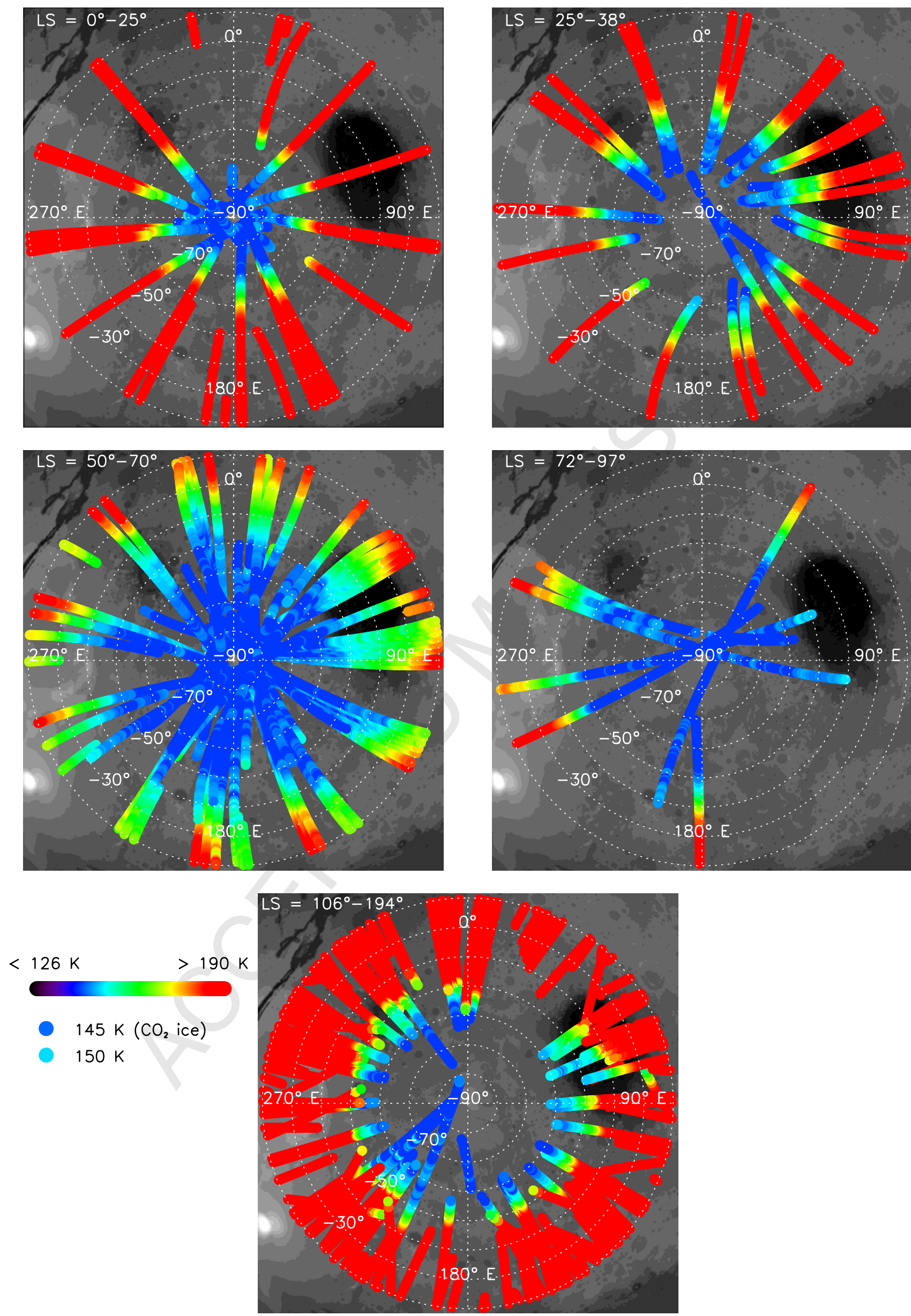


\section{ACCEPTED MANUSCRIPT}
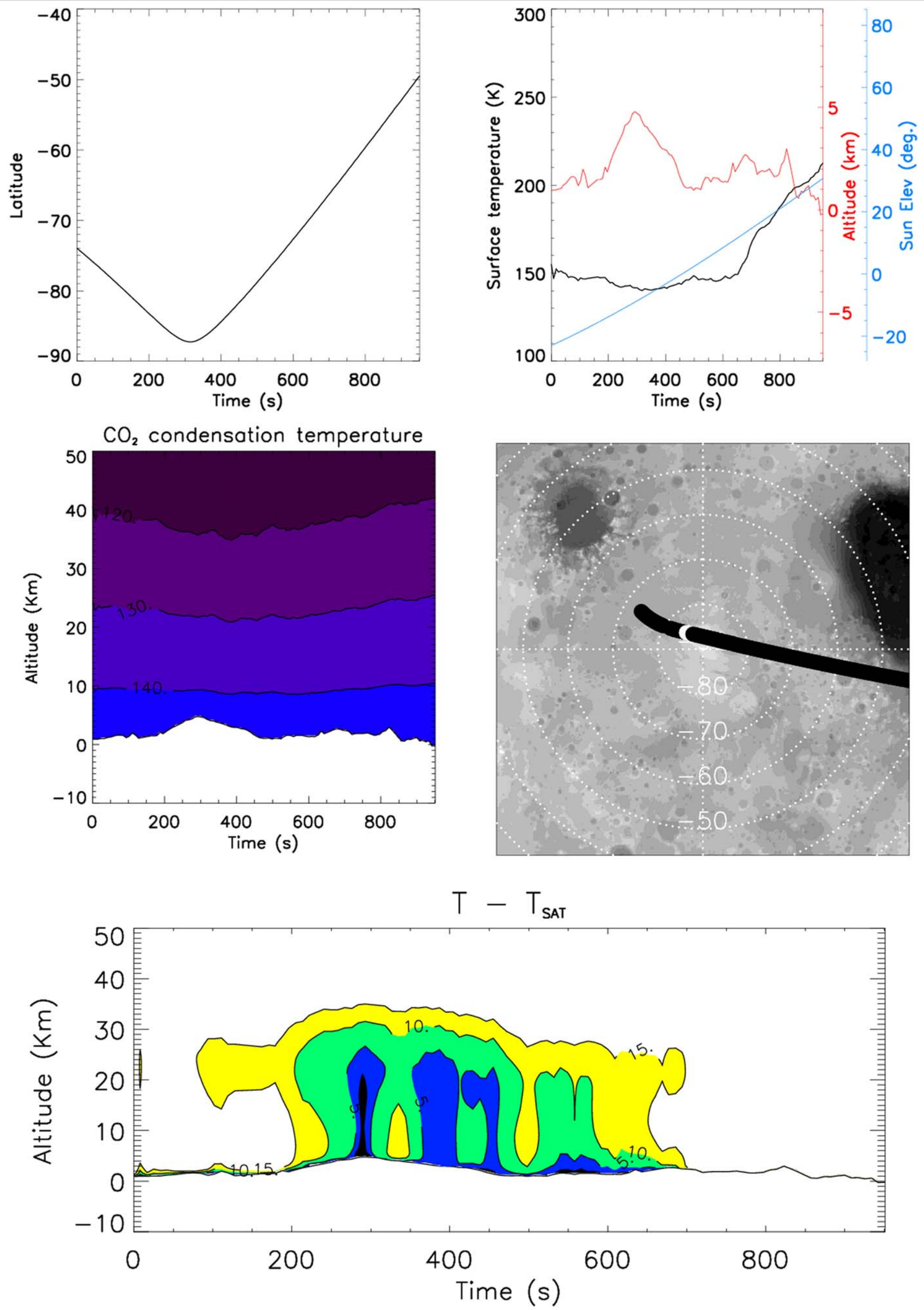


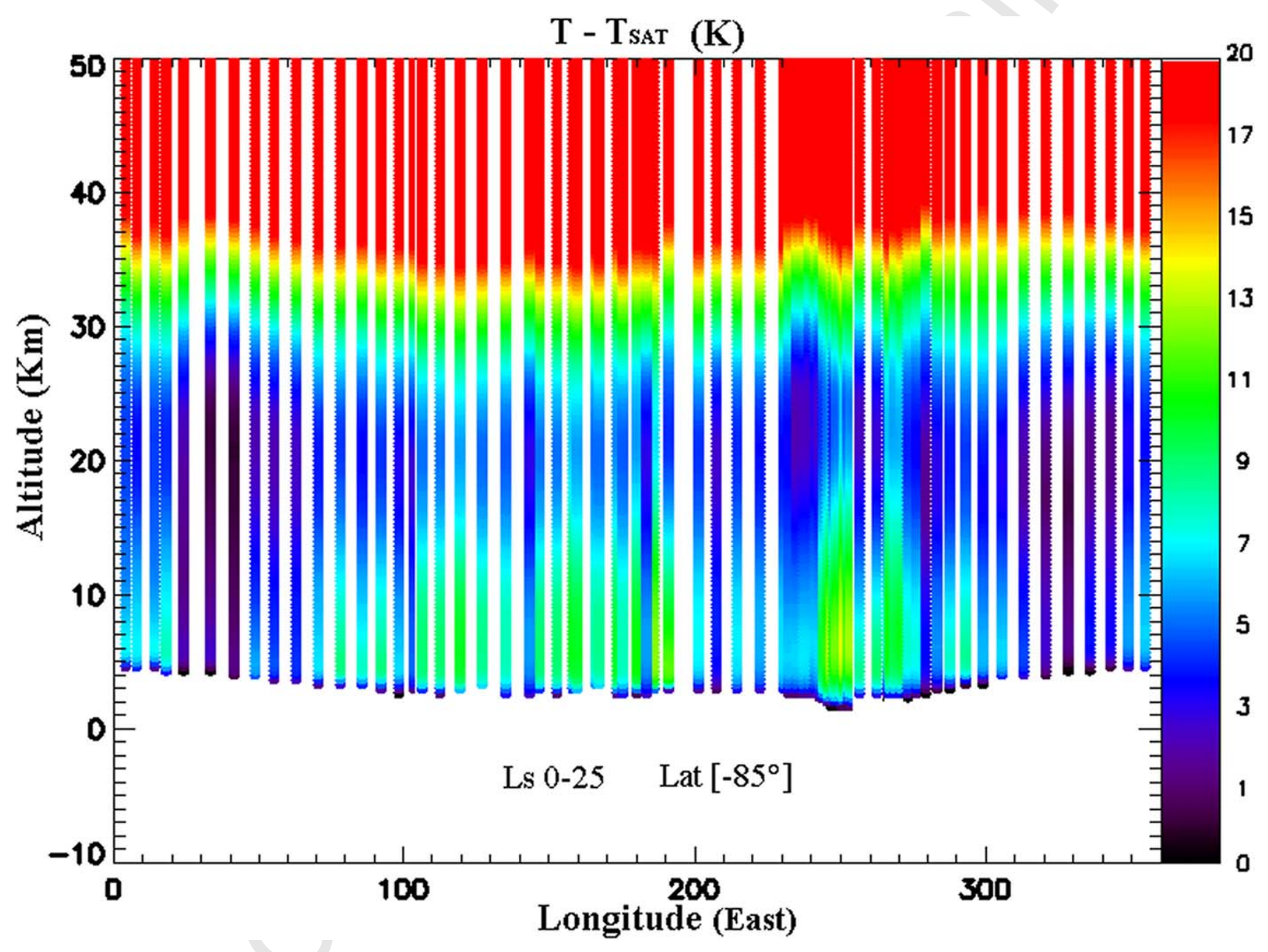




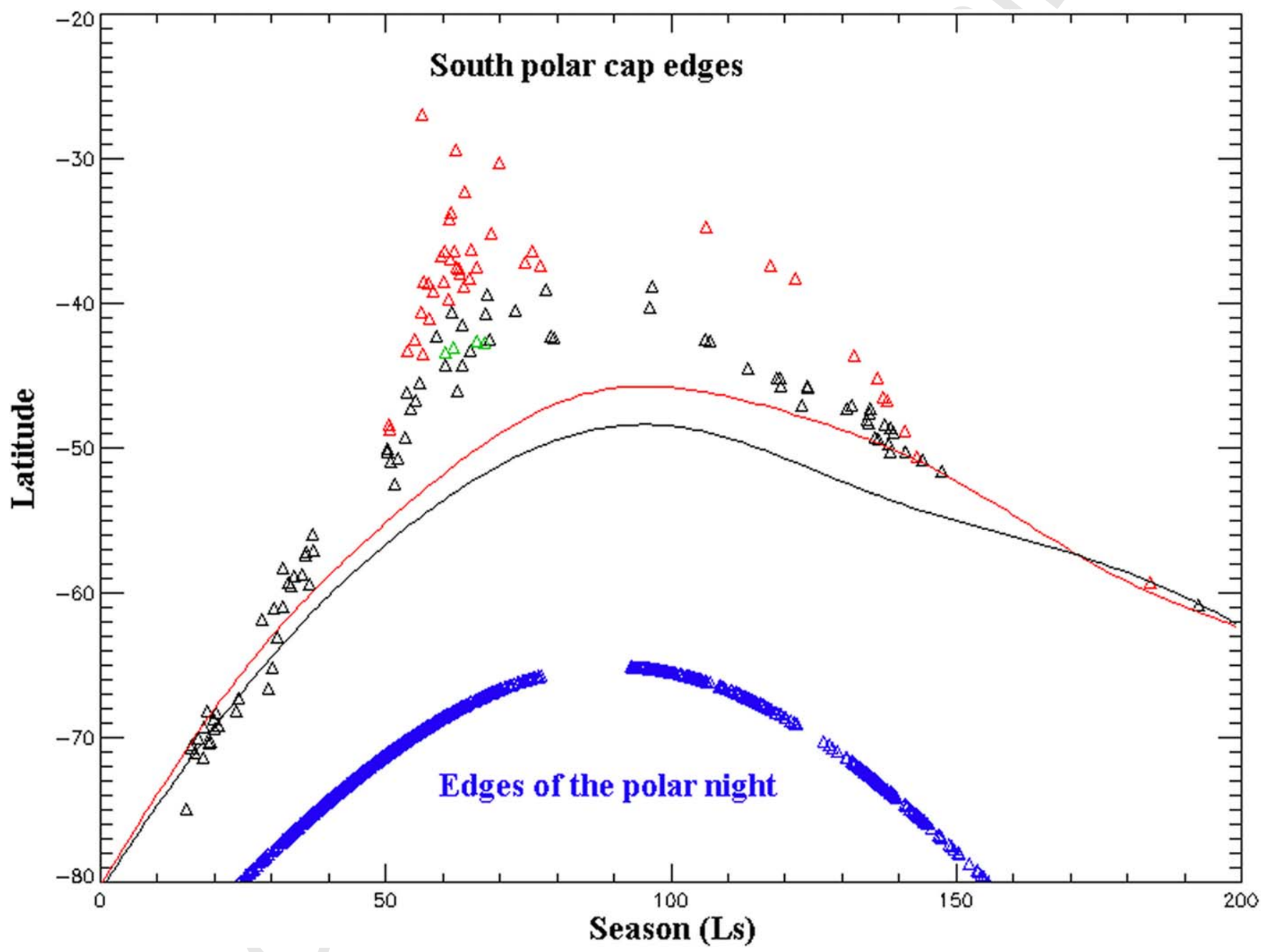




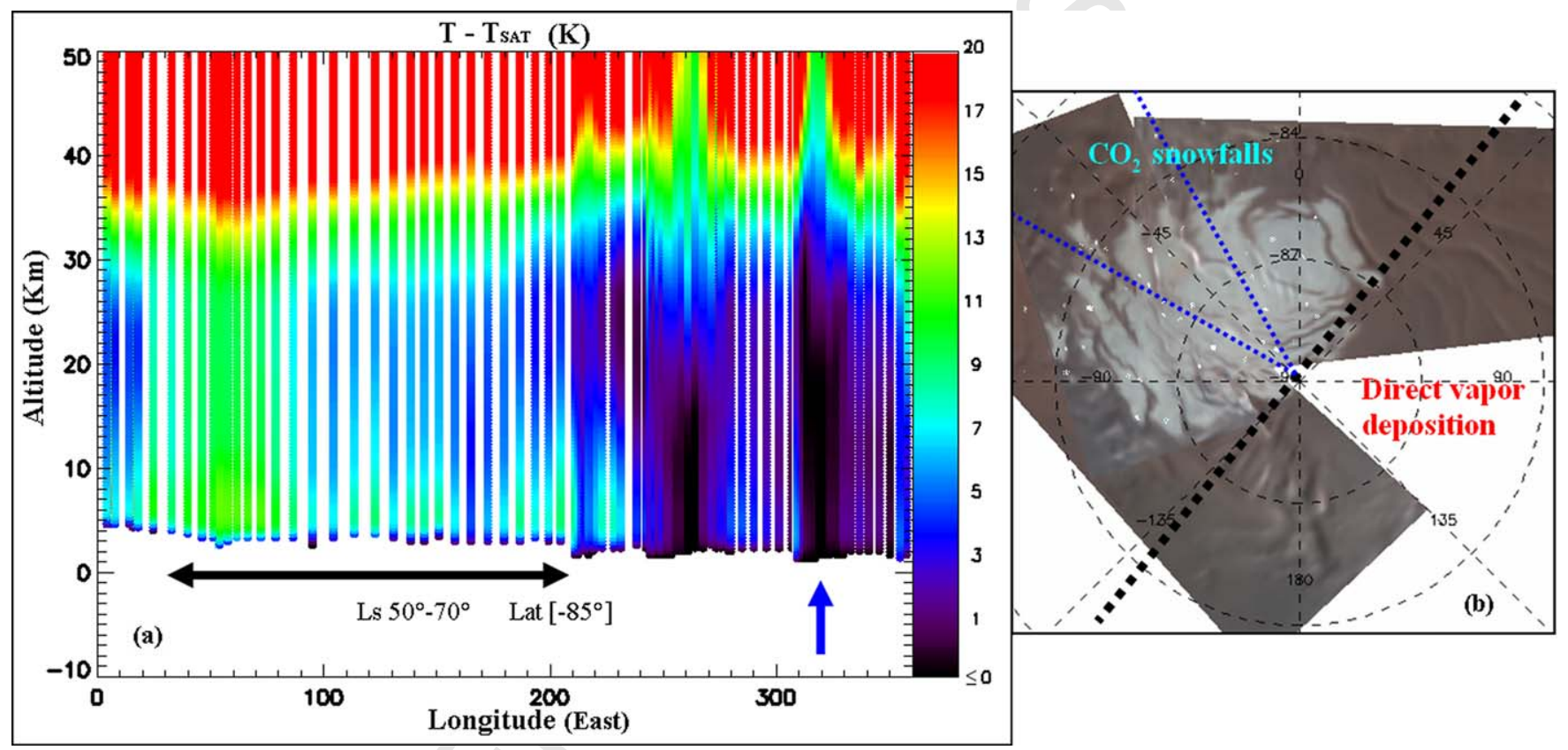




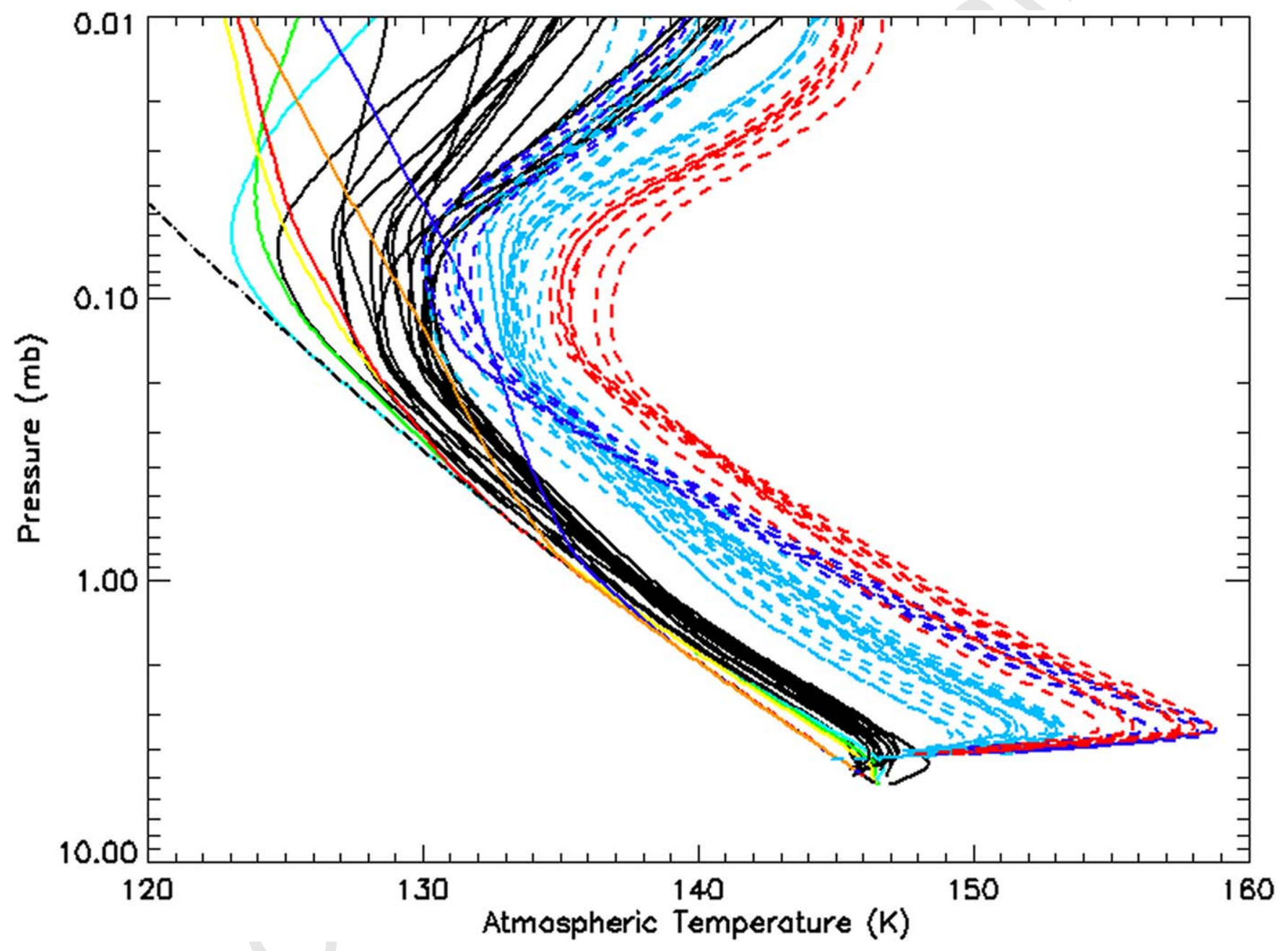




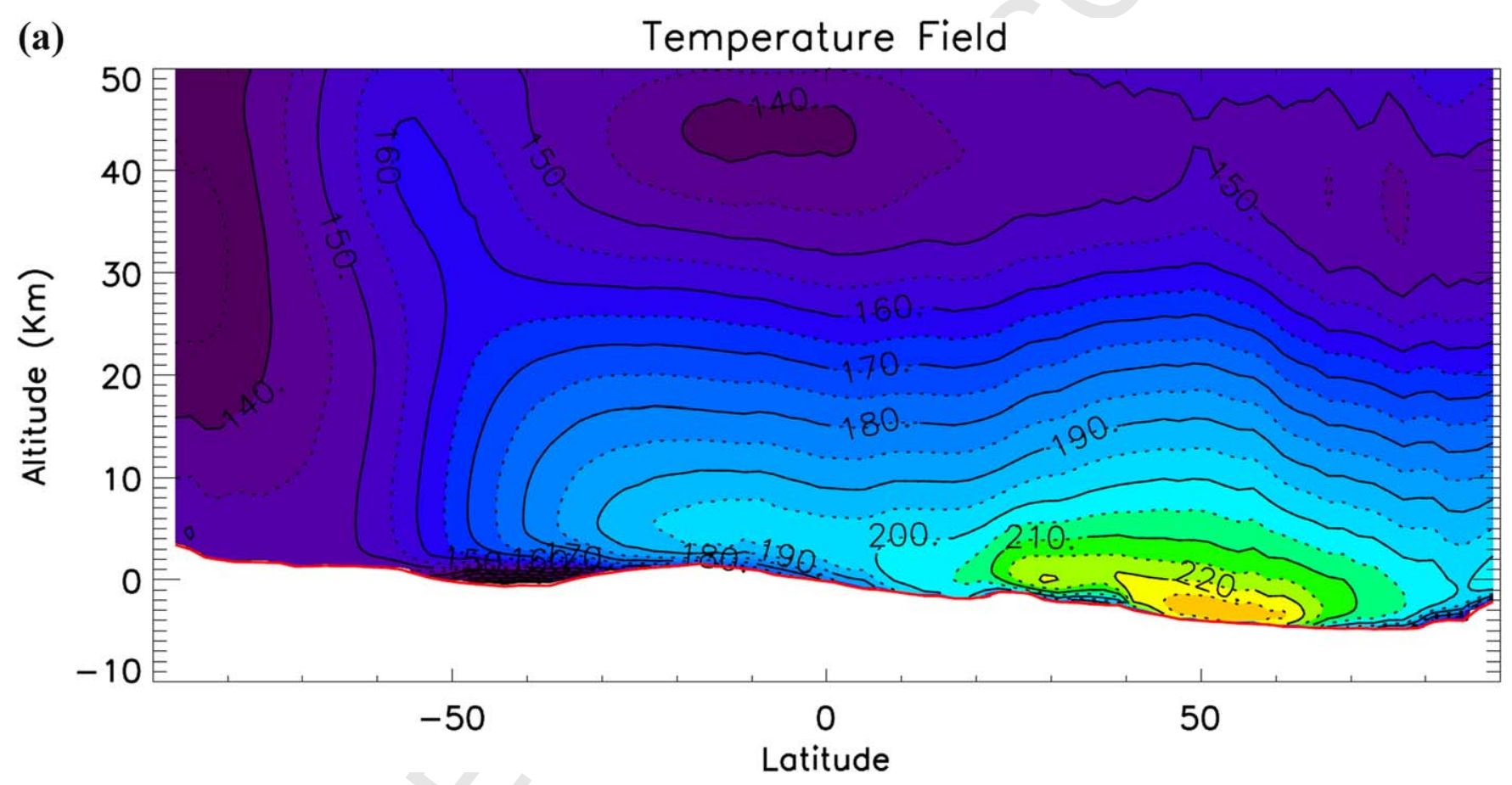


(b) Zonal Wind (m/s). PFS

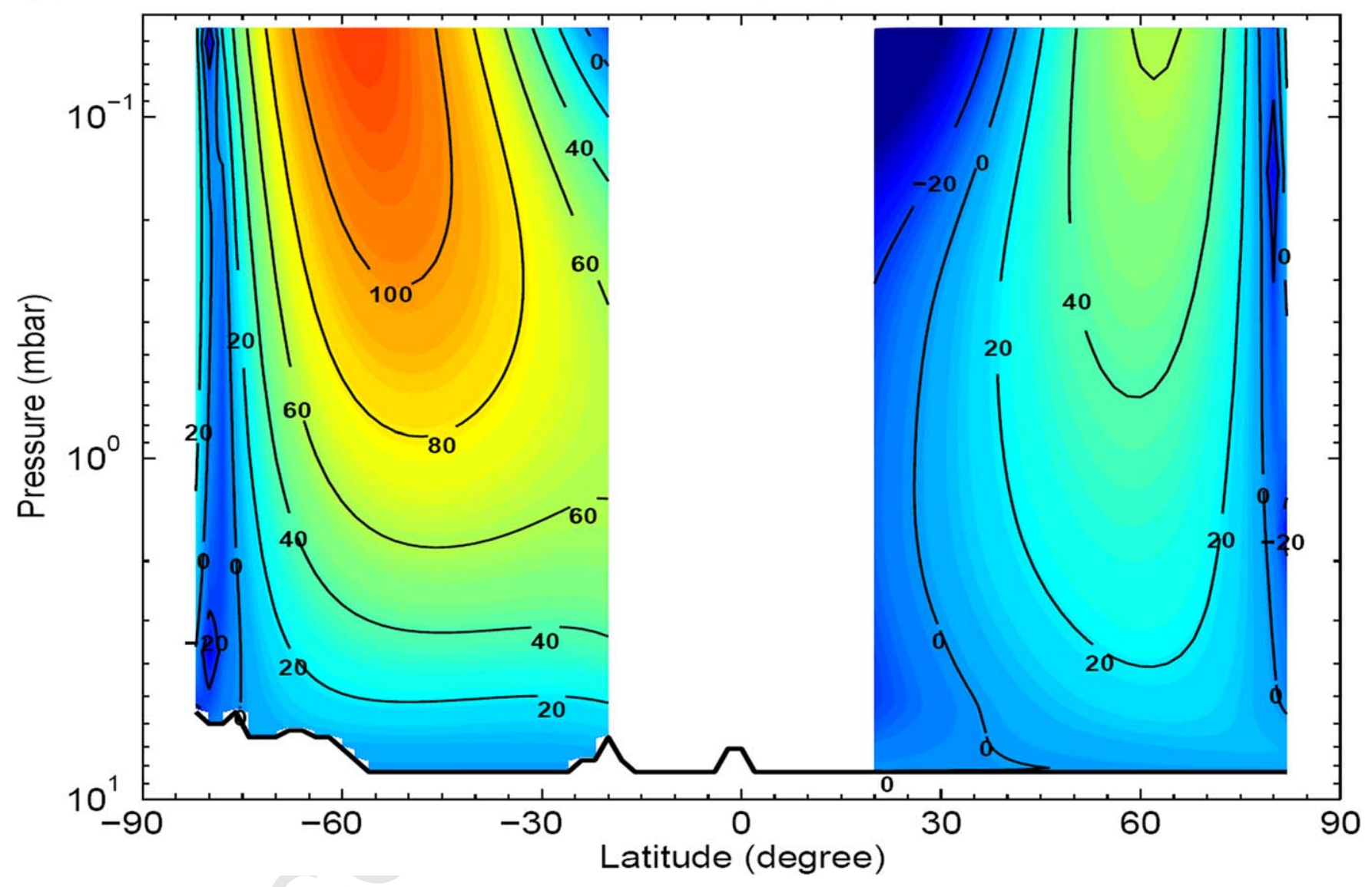




\section{ACCEPTED MANUSCRIPT}

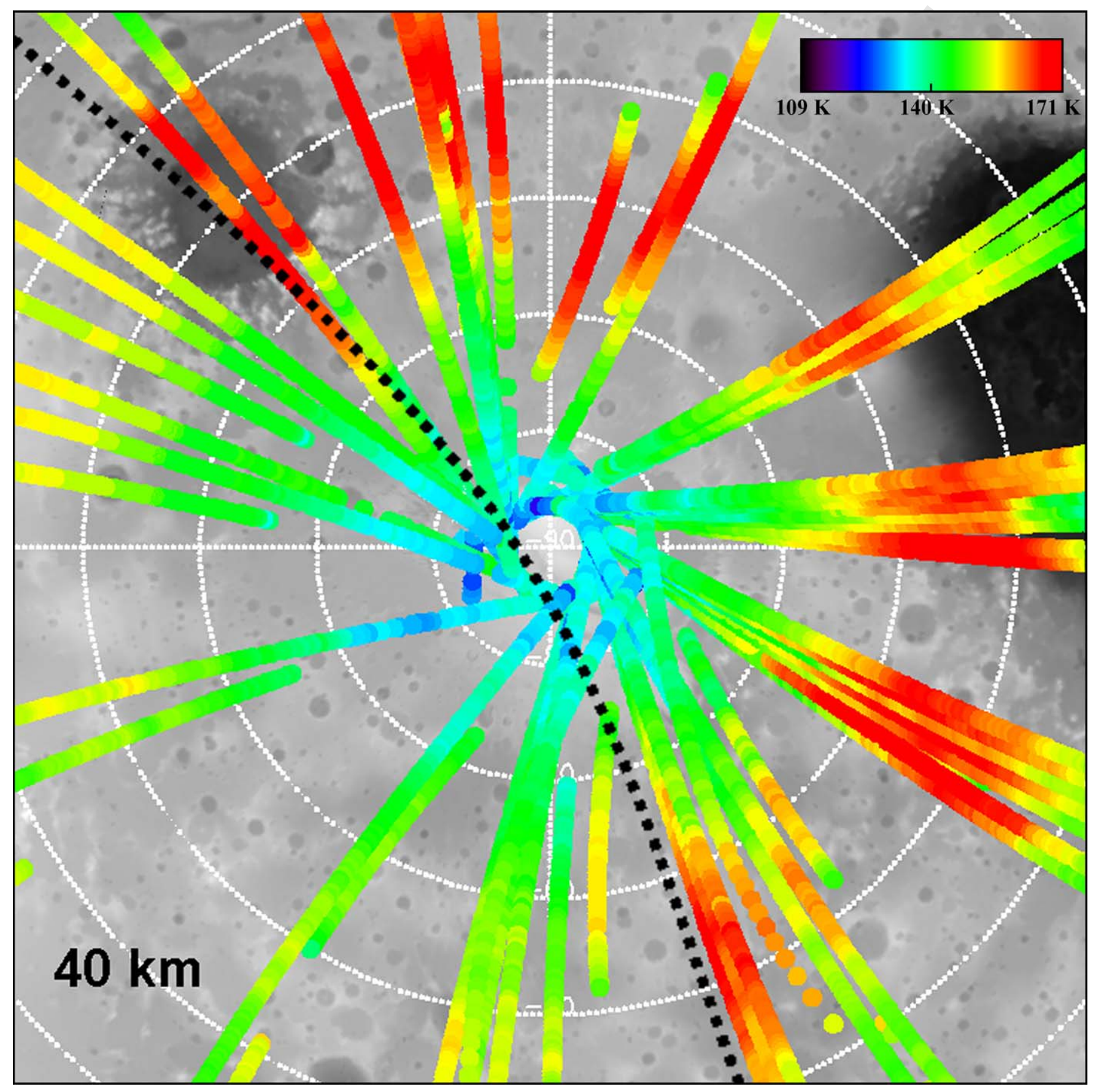




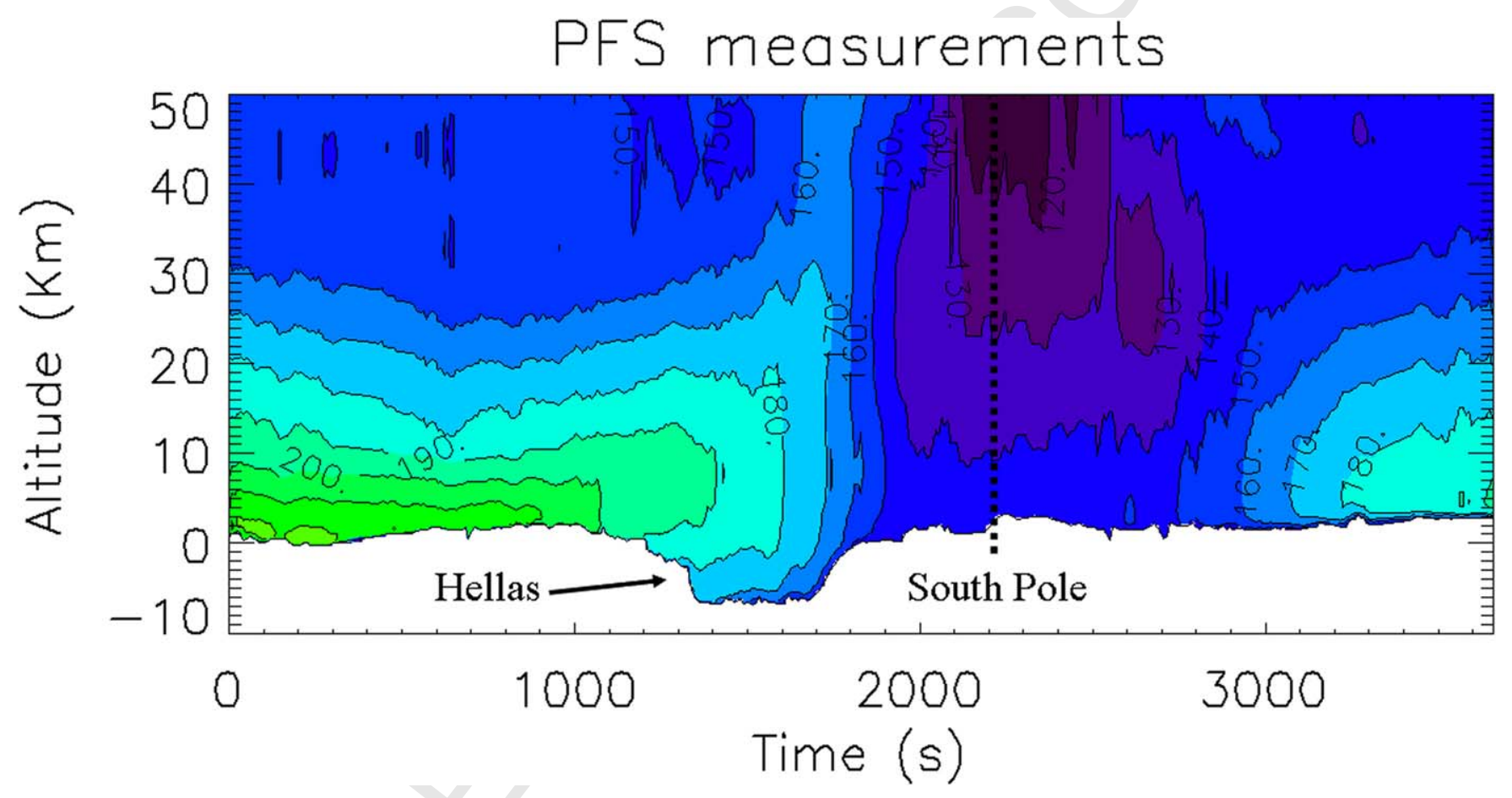




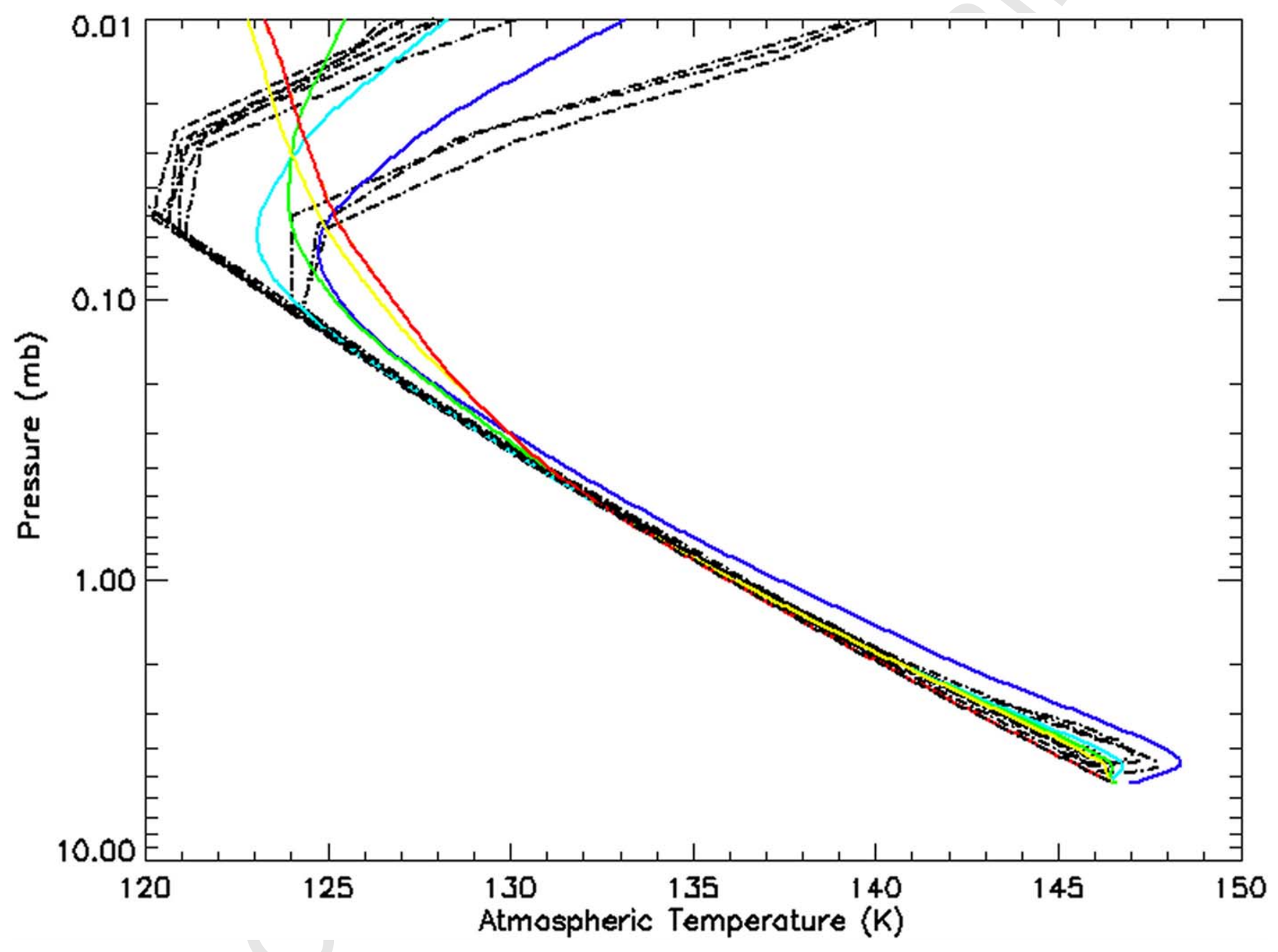




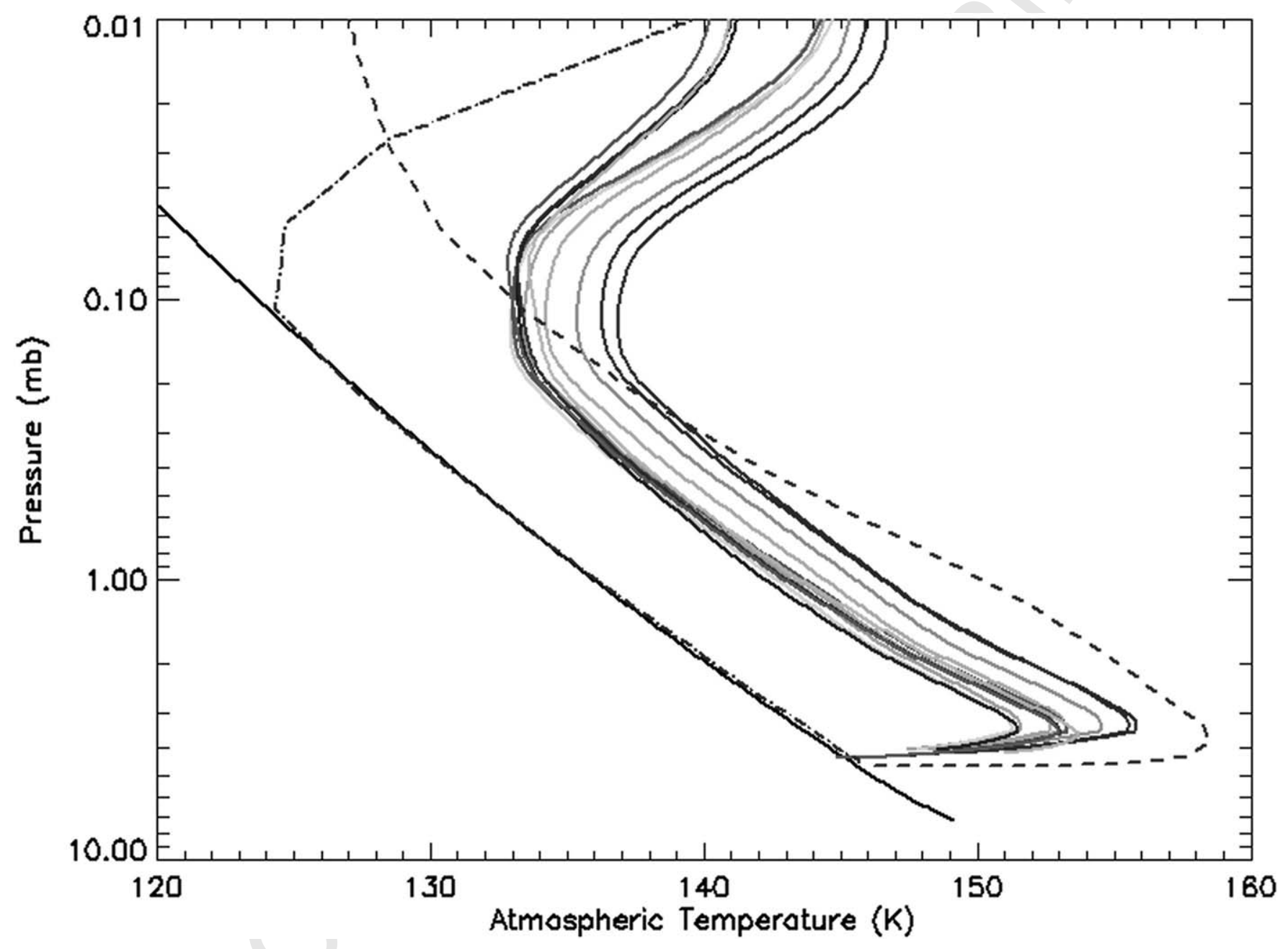




\section{ACCEPTED MANUSCRIPT}
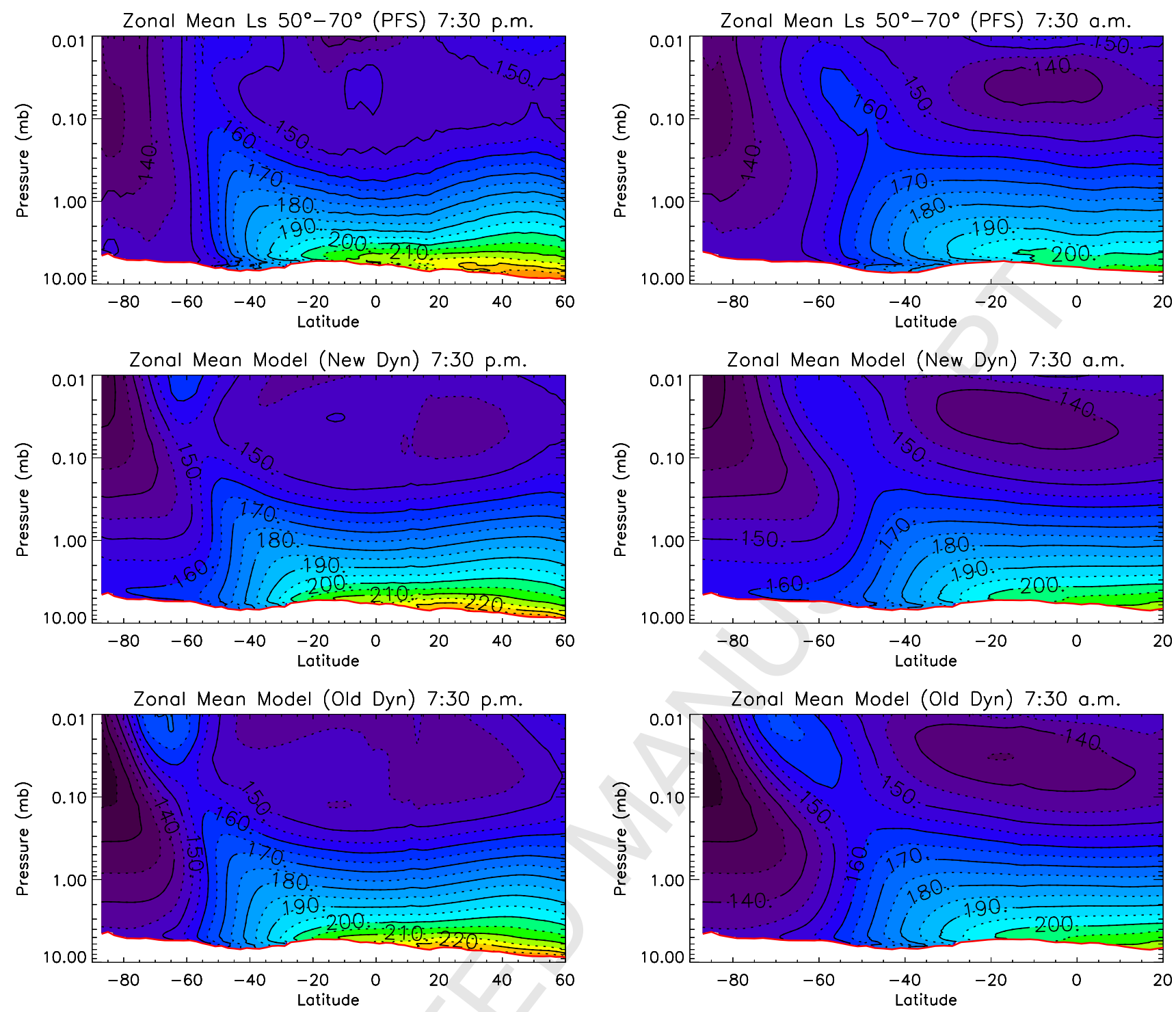

Zonal Mean Ls $50^{\circ}-70^{\circ}$ (PFS - Newdyn) 7:30 p.m.

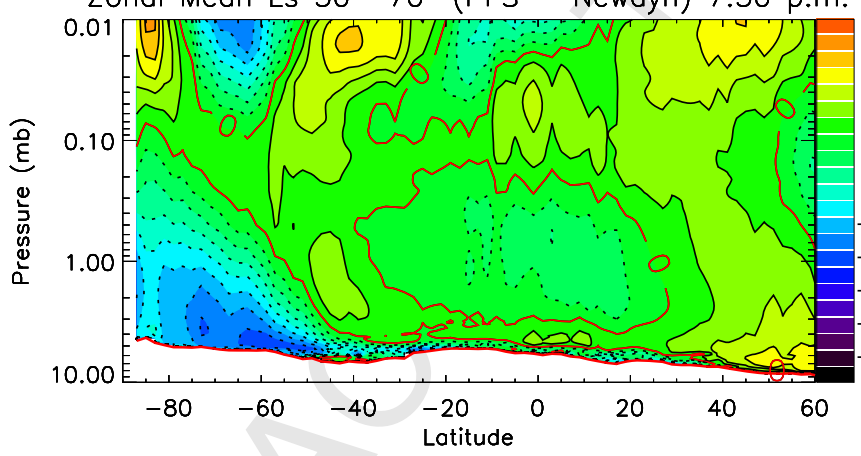

Zonal Mean Ls $50^{\circ}-70^{\circ}$ (PFS - Olddyn) 7:30 p.m.

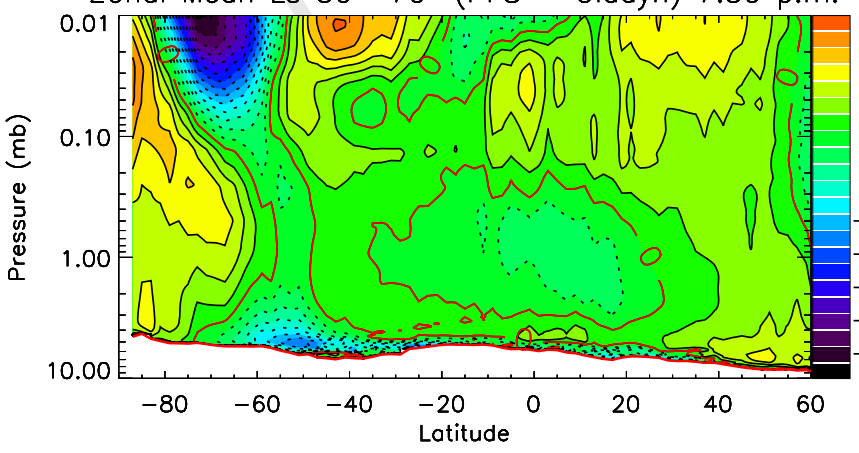

Zonal Mean Ls $50^{\circ}-70^{\circ}$ (PFS - Newdyn) 7:30 a.m.

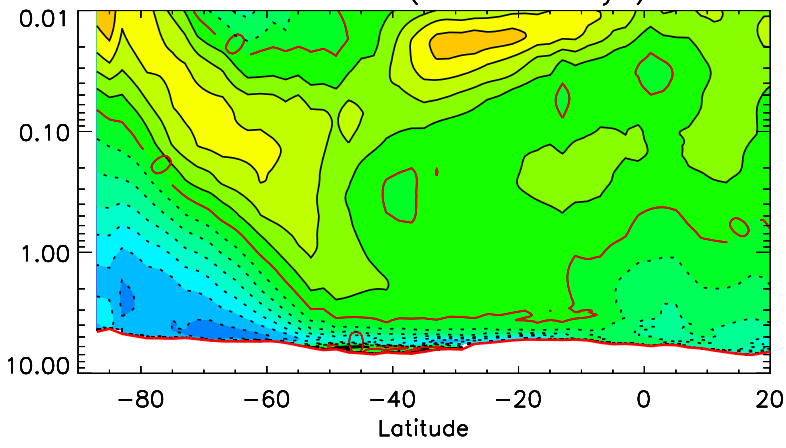

Zonal Mean Ls $50^{\circ}-70^{\circ}$ (PFS - Olddyn) 7:30 a.m.

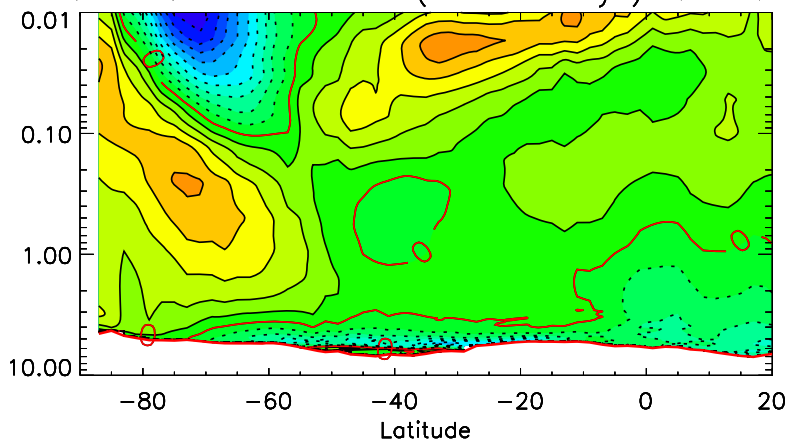




\section{ACCEPTED MANUSCRIPT}
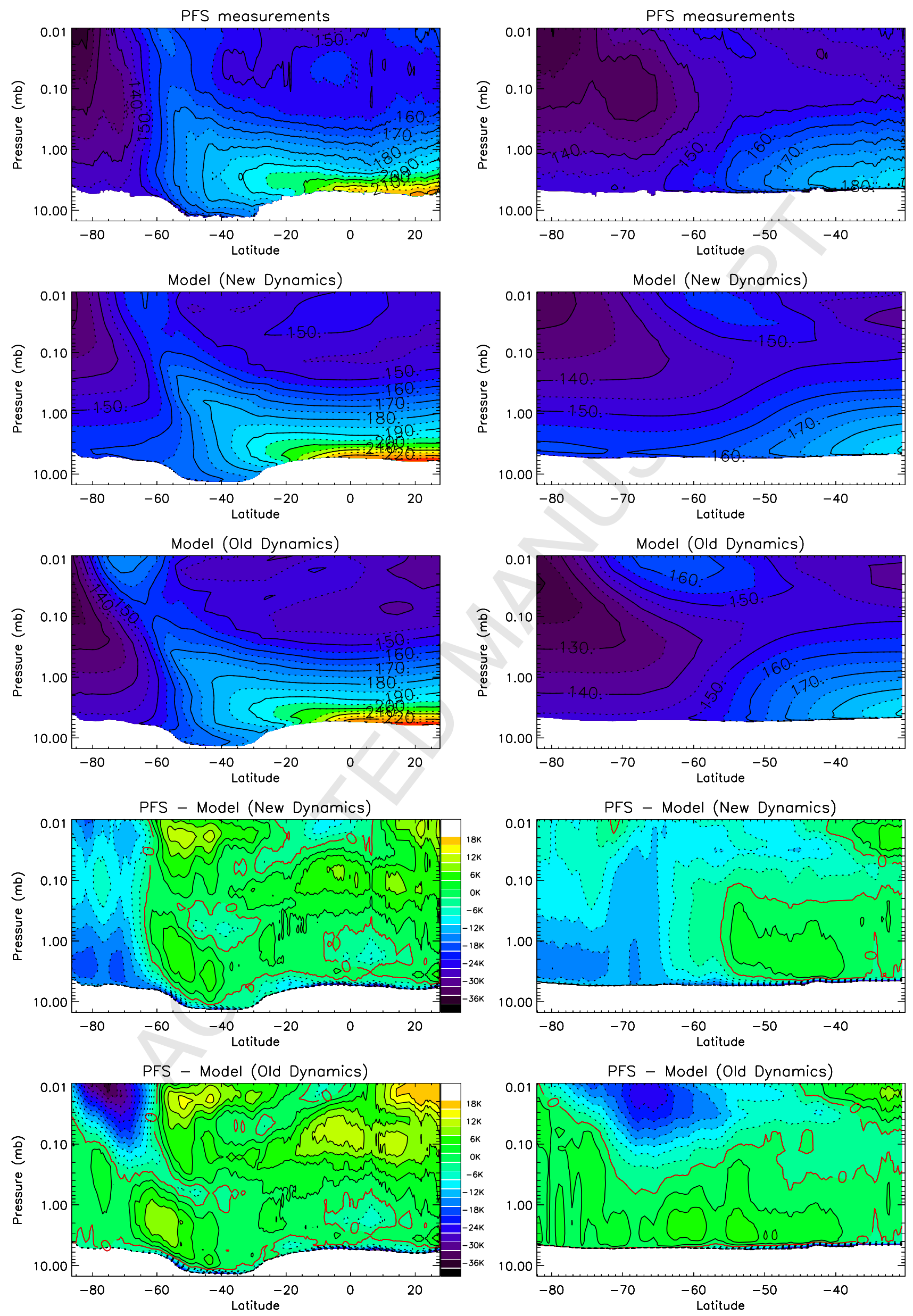

I10324_Fig15 

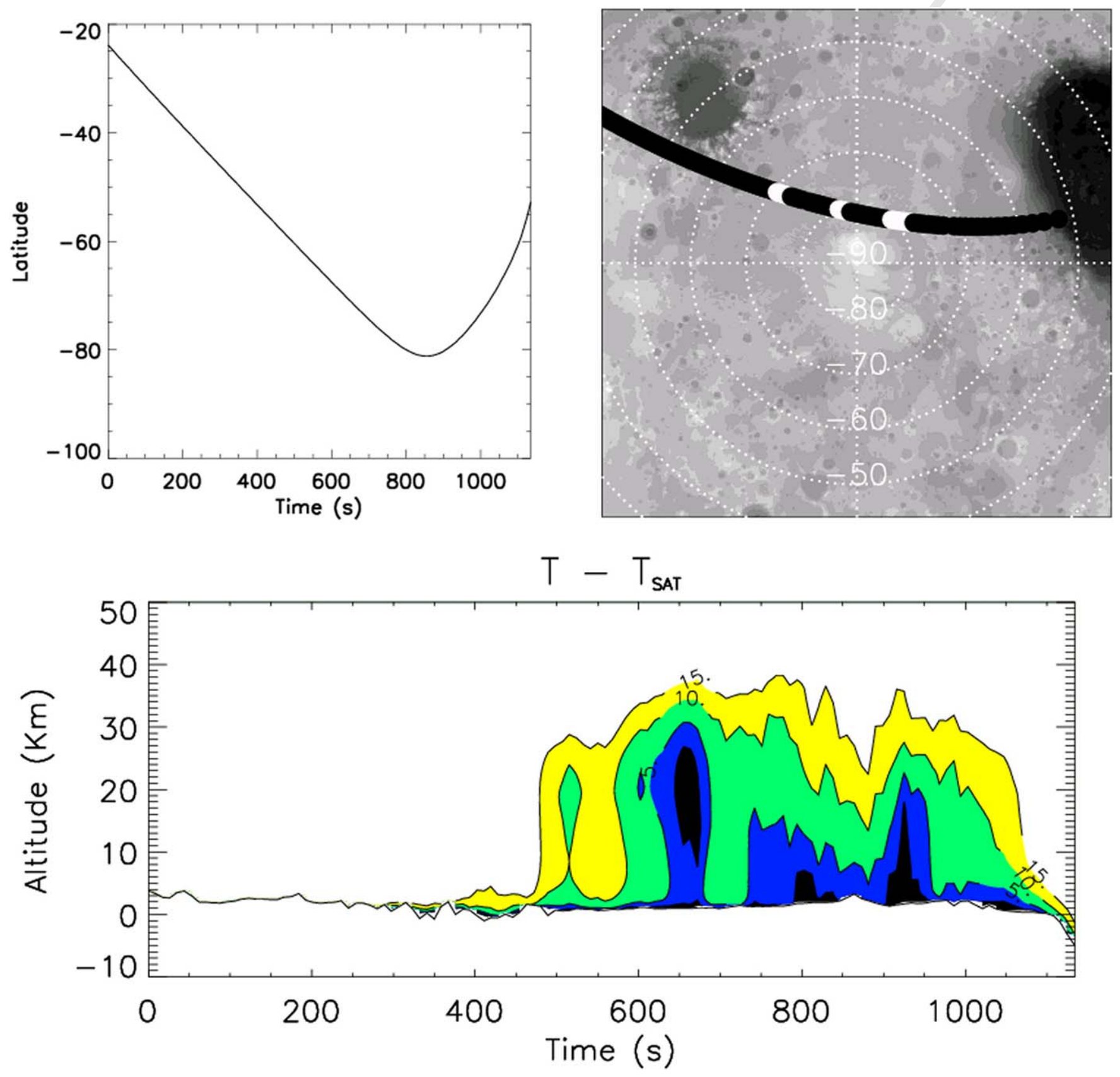